TRANSACTIONS OF THE

AMERICAN MATHEMATICAL SOCIETY

Volume 355, Number 6, Pages 2413-2438

S 0002-9947(03)03235-5

Article electronically published on January 14, 2003

\title{
A POSITIVE RADIAL PRODUCT FORMULA FOR THE DUNKL KERNEL
}

\author{
MARGIT RÖSLER
}

\begin{abstract}
It is an open conjecture that generalized Bessel functions associated with root systems have a positive product formula for nonnegative multiplicity parameters of the associated Dunkl operators. In this paper, a partial result towards this conjecture is proven, namely a positive radial product formula for the non-symmetric counterpart of the generalized Bessel function, the Dunkl kernel. Radial here means that one of the factors in the product formula is replaced by its mean over a sphere. The key to this product formula is a positivity result for the Dunkl-type spherical mean operator. It can also be interpreted in the sense that the Dunkl-type generalized translation of radial functions is positivity-preserving. As an application, we construct Dunkl-type homogeneous Markov processes associated with radial probability distributions.
\end{abstract}

\section{INTRODUCTION}

Along with addition formulas, product formulas have always been a challenging topic in the area of one-variable special functions. Typically, positive product formulas are obvious for particular parameters from a group-theoretical background (when e.g. the functions under consideration have an interpretation as the spherical functions of a Gelfand pair), but it is often difficult to obtain a generalization to larger classes of parameter values. For special functions in several variables such questions seem to be even more intricate. One open conjecture in this direction concerns positive product formulas for multivariable Bessel functions associated with root systems, provided all multiplicity parameters are nonnegative. In the present paper, we prove a partial result towards a positive product formula for multivariable Bessel functions on a Weyl chamber, namely a positive radial product formula for the non-symmetric counterpart of the generalized Bessel function, the Dunkl kernel. This kernel is the analogue of the usual exponential function in the theory of rational Dunkl operators as developed in [6], [7], and [8]. To become more precise, let us briefly introduce our setting. Let $R$ be a (reduced, not necessarily crystallographic) root system in $\mathbb{R}^{N}$, equipped with the standard Euclidean inner product $\langle.,$.$\rangle , i.e., R \subset \mathbb{R}^{N} \backslash\{0\}$ is finite with $R \cap \mathbb{R} \alpha=\{ \pm \alpha\}$ and $\sigma_{\alpha}(R)=R$ for all $\alpha \in R$, where $\sigma_{\alpha}$ denotes the reflection in the hyperplane perpendicular to $\alpha$. We assume $R$ to be normalized such that $\langle\alpha, \alpha\rangle=2$ for all $\alpha \in R$. This simplifies formulas, but is no loss of generality for our purposes. Further, let $G$ denote the

Received by the editors October 2, 2002

2000 Mathematics Subject Classification. Primary 33C52; Secondary 44A35, 35L15.

Key words and phrases. Dunkl operators, Dunkl kernel, product formula, multivariable Bessel functions. 
finite reflection group generated by $\left\{\sigma_{\alpha}, \alpha \in R\right\}$ and let $k: R \rightarrow \mathbb{C}$ be a fixed multiplicity function on $R$, i.e., a function that is constant on the orbits under the action of $G$. We shall always assume that $k$ is nonnegative, i.e., $k(\alpha) \geq 0$ for all $\alpha \in R$. The first-order rational Dunkl operators attached to $G$ and $k$ are defined by

$$
T_{\xi}(k) f(x)=\partial_{\xi} f(x)+\sum_{\alpha \in R_{+}} k(\alpha)\langle\alpha, \xi\rangle \frac{f(x)-f\left(\sigma_{\alpha} x\right)}{\langle\alpha, x\rangle}, \quad x, \xi \in \mathbb{R}^{N} .
$$

Here $\partial_{\xi}$ denotes the derivative in the direction $\xi$ and $R_{+}$is some fixed positive subsystem of $R$. The definition is independent of the special choice of $R_{+}$, thanks to the $G$-invariance of $k$. As first shown in [6], the $T_{\xi}(k), \xi \in \mathbb{R}^{N}$, generate a commutative algebra of differential-reflection operators. This is the foundation for rich analytic structures related to them. In particular, there exist a counterpart of the usual exponential function, called the Dunkl kernel, and an analogue of the Euclidean Fourier transform with respect to this kernel. The Dunkl kernel $E_{k}$ is holomorphic on $\mathbb{C}^{N} \times \mathbb{C}^{N}$ and symmetric in its arguments. Similarly to the situation for spherical functions on a Riemannian symmetric space, the function $E_{k}(., y)$ with fixed spectral parameter $y \in \mathbb{C}^{N}$ may be characterized as the unique analytic solution of the joint eigenvalue problem

$$
T_{\xi}(k) f=\langle\xi, y\rangle f \quad \text { for all } \xi \in \mathbb{C}^{N}, f(0)=1,
$$

cf. [20. Apart from the trivial case $k=0$, where $E_{k}(x, y)=e^{\langle x, y\rangle}$, the kernel $E_{k}$ is explicitly known in only a very few cases. These include the rank-one case (see Section [2.3) as well as the symmetric group $G=S_{3}([9)$. The reflection-invariant counterpart of $E_{k}$ is the so-called generalized Bessel function

$$
J_{k}(x, y)=\frac{1}{|G|} \sum_{g \in G} E_{k}(g x, y),
$$

which was first studied in 20. It is $G$-invariant in both arguments, and is naturally considered on the Weyl chambers of $G$. If the rank of $R$ is one, then $J_{k}$ coincides with a usual Bessel function. In particular cases, all related to Weyl groups and certain half-integer multiplicity parameters, generalized Bessel functions can be given an interpretation as the spherical functions of a Euclidean-type symmetric space. We shall take a closer look at these examples in the Appendix, Section 7 . In these cases, and for all nonnegative multiplicity parameters in the rank-one case, the generalized Bessel functions $J_{k}$ have a positive product formula of the form

$$
J_{k}(x, z) J_{k}(y, z)=\int_{\bar{C}} J_{k}(\xi, z) d \nu_{x, y}^{k}(\xi) \quad \text { for all } z \in \mathbb{C}^{N} .
$$

Here $\bar{C}$ is the topological closure of the Weyl chamber

$$
C=\left\{x \in \mathbb{R}^{N}:\langle\alpha, x\rangle>0 \text { for all } \alpha \in R_{+}\right\},
$$

and the $\nu_{x, y}^{k}$ are compactly supported probability measures on $\bar{C}$. It is conjectured that a positive product formula of the form (1.3) should in fact be valid for arbitrary reflection groups and nonnegative multiplicity parameters. In that case, one would obtain a positivity-preserving convolution of regular bounded Borel measures on the chamber $\bar{C}$ when defining the convolution of point measures according to $\delta_{x} \circ_{k} \delta_{y}:=\nu_{x, y}^{k}$. In the special cases mentioned above, this convolution induces the structure of a commutative hypergroup on $\bar{C}$, and we conjecture that 
this should be true in general (for nonnegative multiplicities). Roughly speaking, a hypergroup consists of a locally compact Hausdorff space $H$ together with a positivity-preserving convolution on the space of regular bounded Borel measures on $H$, which allows one to carry over the harmonic analysis on a locally compact group to a large extent. The hypergroup setting in particular includes double coset spaces $G / / H$ with $H$ a compact subgroup of a locally compact group $G$. For an introduction to the subject, the reader is referred to [5] or [17.

As already indicated, the generalized exponential function $E_{k}$ is of particular interest, since it gives rise to an integral transform on $\mathbb{R}^{N}$, commonly called the Dunkl transform. On suitable function spaces, this transform maps Dunkl operators to multiplication operators. The Dunkl transform provides a natural generalization of the usual Euclidean Fourier transform, to which it reduces in case $k=0$. It is another open question whether the Dunkl transform admits an interpretation as the Gelfand transform of a suitable $L^{1}$-convolution algebra on $\mathbb{R}^{N}$ for arbitrary parameters $k \geq 0$. Again this is true in the rank-one case. Here the Dunkl kernel satisfies a product formula which leads to a convolution structure on the entire real line, providing a natural extension of the usual group structure, see [21]. In contrast to a hypergroup convolution, this convolution is not positivity-preserving if $k>0$. We conjecture that an analogous statement is true for arbitrary rank and all multiplicities $k \geq 0$. In particular, the Dunkl kernel should have a product formula of the form

$$
E_{k}(x, z) E_{k}(y, z)=\int_{\mathbb{R}^{N}} E_{k}(\xi, z) d \mu_{x, y}^{k}(\xi) \quad \text { for all } z \in \mathbb{C}^{N},
$$

where the measures $\mu_{x, y}^{k}$ are signed Borel measures on $\mathbb{R}^{N}$, which are uniformly bounded with respect to the total variation norm.

This paper presents a result towards both the stated conjectures, namely a positive "radial" product formula for $E_{k}$. More precisely, we shall prove that for each $x \in \mathbb{R}^{N}$ and $t \geq 0$ there exists a unique compactly supported probability measure $\sigma_{x, t}^{k}$ on $\mathbb{R}^{N}$ such that

$$
E_{k}(i x, z) j_{\lambda}(t|z|)=\int_{\mathbb{R}^{N}} E_{k}(i \xi, z) d \sigma_{x, t}^{k}(\xi) \quad \text { for all } z \in \mathbb{R}^{N} .
$$

Here the index of the Bessel function $j_{\lambda}($ see (2.10) $)$ is given by $\lambda=\gamma+N / 2-1$, with

$$
\gamma=\sum_{\alpha \in R_{+}} k(\alpha) \geq 0
$$

Of course, the kernel $E_{k}$ in (1.5) may be equally replaced by the generalized Bessel function $J_{k}$. This product formula will be obtained from a study of the Dunkl-type spherical mean operator, as first introduced in [19. In analogy to the classical case, the spherical mean operator $f \mapsto M_{f}$ is defined for $f \in C^{\infty}\left(\mathbb{R}^{N}\right)$ by

$$
M_{f}(x, t)=\frac{1}{d_{k}} \int_{S^{N-1}} f\left(x *_{k} t y\right) w_{k}(y) d \sigma(y) \quad\left(x \in \mathbb{R}^{N}, t \geq 0\right) .
$$

Here $d_{k}$ is a normalization constant, $w_{k}$ is the $G$-invariant weight function

$$
w_{k}(x)=\prod_{\alpha \in R_{+}}|\langle\alpha, x\rangle|^{2 k(\alpha)} \quad\left(x \in \mathbb{R}^{N}\right),
$$


and $f\left(x *_{k} y\right)$ denotes the Dunkl-type generalized translation, which coincides with the usual group translation on $\mathbb{R}^{N}$ in case $k=0$ and satisfies

$$
E_{k}\left(x *_{k} y, z\right)=E_{K}(x, z) E_{k}(y, z) \text { for all } x, y \in \mathbb{R}^{N}, z \in \mathbb{C}^{N} .
$$

A key result of the present paper states that for $k \geq 0$, the associated spherical mean operator is positivity-preserving. This implies the existence of compactly supported probability measures $\sigma_{x, t}^{k}$ on $\mathbb{R}^{N}$ which represent this operator in the sense that

$$
M_{f}(x, t)=\int_{\mathbb{R}^{N}} f d \sigma_{x, t}^{k} \quad \text { for all } f \in C^{\infty}\left(\mathbb{R}^{N}\right) .
$$

When specializing to $f(x)=E_{k}(i x, z)$ with $z \in \mathbb{R}^{N}$, one obtains (1.5). Similar to the classical case $k=0, M_{f}$ satisfies a second-order differential-reflection equation of Darboux type. A study of the domain of dependence for this equation allows one to deduce further information on the support of the representing measures $\sigma_{x, t}^{k}$. In fact, the support of $\sigma_{x, t}^{k}$ turns out to be contained in the union of closed balls with radius $t$ around the points $g x, g \in G$. In contrast to the classical case, where the support reduces to the sphere $\left\{\xi \in \mathbb{R}^{N}:|\xi-x|=t\right\}$, now full balls as well as the complete $G$-orbit of $x$ have to be taken into account. This is due to the reflection parts in the generalized Darboux equation for $M_{f}$. These results are contained in Theorem 4.1. A slightly weaker variant of Theorem 4.1 is given in Theorem 5.1. Here it is shown that the Dunkl-type generalized translation (more precisely, the mapping $f \mapsto f\left(x *_{k} y\right)$ ) is positivity-preserving when restricted to radial $f$. In Section [6] the results are applied to construct "radial" semigroups $\left(P_{t}\right)_{t \geq 0}$ of Markov kernels on $\mathbb{R}^{N}$, which are translation-invariant in the generalized sense of 24. Here $P_{t}$ is obtained by a Dunkl-type translation of a probability measure $\mu_{t}$ of the form $d \mu_{t}(x)=w_{k}(x) d \mu_{t}^{\prime}(x)$, where $\mu_{t}^{\prime}$ is rotation-invariant.

\section{Preliminaries}

In this introductory part we give an account on results from Dunkl theory which will be relevant for the sequel. These concern in particular the Dunkl kernel, the Dunkl transform and generalized translations. We also include a discussion of the rank-one case as a motivating example. For a further background in Dunkl theory, the reader is referred to 6], 7], 8], [15, 23, [28, and [10. Concerning root systems and reflection groups, see [14.

Throughout the paper, $\langle.,$.$\rangle denotes the standard Euclidean scalar product in$ $\mathbb{R}^{N}$ as well as its bilinear extension to $\mathbb{C}^{N} \times \mathbb{C}^{N}$. For $x \in \mathbb{R}^{N}$, we write $|x|=\sqrt{\langle x, x\rangle}$. Further, $\mathbb{Z}_{+}:=\{0,1,2, \ldots\}$, and $\mathbb{R}_{+}:=[0, \infty)$. We denote by $C^{\infty}\left(\mathbb{R}^{N}\right)$ the space of infinitely differentiable functions on $\mathbb{R}^{N}$ and by $\mathcal{S}\left(\mathbb{R}^{N}\right)$ the Schwartz space of rapidly decreasing functions, both equipped with the usual Fréchet space topologies. For a locally compact Hausdorff space $X, C_{b}(X)$ denotes the space of continuous, bounded functions on $X$. Further, $M_{b}(X), M_{b}^{+}(X)$ and $M^{1}(X)$ stand for the spaces of regular bounded complex Borel measures on $X$, those which are nonnegative, and those which are probability measures, respectively. The $\sigma\left(M_{b}(X), C_{b}(X)\right)$-topology is referred to as the weak topolgy on $M_{b}(X)$, and the $\sigma$-algebra of Borel sets in $X$ is denoted by $\mathcal{B}(X)$.

2.1. Basics from Dunkl theory. Let $G$ be a finite reflection group on $\mathbb{R}^{N}$ with root system $R$, and fix a positive subsystem $R_{+}$of $R$ as well as a nonnegative multiplicity function $k$. The associated Dunkl operators $T_{\xi}(k)$, defined according 
to (1.1), share many properties with usual partial derivatives. In particular, if $f \in C^{k}\left(\mathbb{R}^{N}\right)$, then $T_{\xi}(k) f \in C^{k-1}\left(\mathbb{R}^{N}\right)$, the $T_{\xi}(k)$ are homogeneous of degree -1 on polynomials, and they leave the Schwartz space $\mathcal{S}\left(\mathbb{R}^{N}\right)$ invariant. Moreover, $T_{\xi}(k)$ is $G$-equivariant:

$$
g \circ T_{\xi}(k) \circ g^{-1}=T_{g \xi}(k) \quad(g \in G) .
$$

The counterpart of the usual Laplacian is the Dunkl Laplacian, defined by

$$
\Delta_{k}:=\sum_{i=1}^{N} T_{\xi_{i}}(k)^{2},
$$

where $\left\{\xi_{i}, i=1, \ldots, N\right\}$ is an arbitrary orthonormal basis of $\left(\mathbb{R}^{N},\langle.,\rangle.\right)$, cf. [6]. It is given explicitly by

$$
\Delta_{k} f(x)=L_{k} f(x)-2 \sum_{\alpha \in R_{+}} k(\alpha) \frac{f(x)-f\left(\sigma_{\alpha} x\right)}{\langle\alpha, x\rangle^{2}},
$$

with the singular elliptic operator

$$
L_{k} f(x):=\Delta f(x)+2 \sum_{\alpha \in R_{+}} k(\alpha) \frac{\langle\nabla f(x), \alpha\rangle}{\langle\alpha, x\rangle} .
$$

According to [7], there exists a unique degree-of-homogeneity-preserving linear isomorphism $V_{k}$ on polynomials such that

$$
T_{\xi}(k) V_{k}=V_{k} \partial_{\xi} \text { for all } \xi \in \mathbb{R}^{N} \text { and } V_{k}(1)=1 .
$$

It is shown in 23] that $V_{k}$ has a Laplace-type representation of the form

$$
V_{k} f(x)=\int_{\mathbb{R}^{N}} f(\xi) d \mu_{x}^{k}(\xi)
$$

with a unique probability measure $\mu_{x}^{k} \in M^{1}\left(\mathbb{R}^{N}\right)$ whose support is contained in

$$
C(x):=\operatorname{co}\{g x, g \in G\},
$$

the convex hull of the $G$-orbit of $x$ in $\mathbb{R}^{N}$. By means of formula (2.2), $V_{k}$ may be extended to various larger function spaces, including $C^{\infty}\left(\mathbb{R}^{N}\right)$. We denote this extension by $V_{k}$ again. In fact, $V_{k}$ establishes a homeomorphism of $C^{\infty}\left(\mathbb{R}^{N}\right)$; see Theorem 4.6 of [16] or [28].

The Dunkl kernel asociated with $G$ and $k$ is defined by

$$
E_{k}(x, y)=V_{k}\left(e^{\langle\cdot, y\rangle}\right)(x)=\int_{\mathbb{R}^{N}} e^{\langle\xi, y\rangle} d \mu_{x}^{k}(\xi) \quad\left(x \in \mathbb{R}^{N}, y \in \mathbb{C}^{N}\right) .
$$

For fixed $y, E_{k}(., y)$ is the unique real-analytic solution of (1.2), cf. 20. The kernel $E_{k}$ is symmetric in its arguments and has a unique holomorphic extension to $\mathbb{C}^{N} \times \mathbb{C}^{N}$. Moreover,

$$
E_{k}(\lambda z, w)=E_{k}(z, \lambda w) \quad \text { and } \quad E_{k}(g z, g w)=E_{k}(z, w)
$$

for all $z, w \in \mathbb{C}^{N}, \lambda \in \mathbb{C}$ and $g \in G$. Let $w_{k}$ denote the $G$-invariant weight function (1.6). The associated Dunkl transform on $L^{1}\left(\mathbb{R}^{N}, w_{k}\right)$ is then defined by

$$
\widehat{f}^{k}(\xi):=c_{k}^{-1} \int_{\mathbb{R}^{N}} f(x) E_{k}(-i \xi, x) w_{k}(x) d x \quad\left(\xi \in \mathbb{R}^{N}\right) .
$$


Here $c_{k}$ denotes the Mehta-type constant

$$
c_{k}:=\int_{\mathbb{R}^{N}} e^{-|x|^{2} / 2} w_{k}(x) d x .
$$

We shall also consider the Dunkl transform on the measure space $M_{b}\left(\mathbb{R}^{N}\right)$,

$$
\widehat{\mu}^{k}(\xi):=\int_{\mathbb{R}^{N}} E_{k}(-i \xi, x) d \mu(x) \quad\left(\xi \in \mathbb{R}^{N}\right) .
$$

Many properties of the Euclidean Fourier transform carry over to the Dunkl transform. The results listed below can be found in [8], [15] and 24].

\section{Proposition 2.1.}

(1) The Dunkl transform $f \mapsto \widehat{f}^{k}$ is a homeomorphism of $\mathcal{S}\left(\mathbb{R}^{N}\right)$. Its inverse is given by $f^{\vee k}(\xi):=\widehat{f}^{k}(-\xi)$.

(2) ( $L^{1}$-inversion) If $f \in L^{1}\left(\mathbb{R}^{N}, w_{k}\right)$ with $\widehat{f}^{k} \in L^{1}\left(\mathbb{R}^{N}, w_{k}\right)$, then $f=\left(\widehat{f}^{k}\right)^{\vee k}$ a.e.

(3) (Plancherel Theorem) The Dunkl transform on $\mathcal{S}\left(\mathbb{R}^{N}\right)$ extends uniquely to an isometric isomorphism of $L^{2}\left(\mathbb{R}^{N}, w_{k}\right)$.

(4) The Dunkl transform is injective on $M_{b}\left(\mathbb{R}^{N}\right)$.

(5) (Lévy's continuity theorem) Let $\left(\mu_{n}\right)_{n \in \mathbb{N}} \subset M_{b}^{+}\left(\mathbb{R}^{N}\right)$ be such that $\left(\widehat{\mu}_{n}^{k}\right)_{n \in \mathbb{N}}$ converges pointwise to a function $\varphi: \mathbb{R}^{N} \rightarrow \mathbb{C}$ that is continuous at 0 . Then there exists a unique measure $\mu \in M_{b}^{+}\left(\mathbb{R}^{N}\right)$ with $\widehat{\mu}^{k}=\varphi$, and $\left(\mu_{n}\right)_{n \in \mathbb{N}}$ tends weakly to $\mu$.

In [28, a generalized translation on $C^{\infty}\left(\mathbb{R}^{N}\right)$ is defined by

$$
\tau_{y} f(x):=V_{k}^{x} V_{k}^{y}\left(V_{k}^{-1} f\right)(x+y), \quad x, y \in \mathbb{R}^{N} .
$$

Here the superscript denotes the relevant variable. Notice that

$$
\tau_{0} f=f, \quad T_{\xi}(k) \tau_{y} f=\tau_{y} T_{\xi}(k) f \quad \text { and } \tau_{y} f(x)=\tau_{x} f(y) \quad \text { for all } x, y \in \mathbb{R}^{N} .
$$

We shall frequently use the more suggestive notation

$$
f\left(x *_{k} y\right):=\tau_{y} f(x) .
$$

For $k=0$, one just obtains the usual group translation on $\mathbb{R}^{N}: f\left(x *_{0} y\right)=f(x+y)$. It is also immediate from the definition that

$$
E_{k}\left(x *_{k} y, z\right)=E_{k}(x, z) E_{k}(y, z) \text { for all } z \in \mathbb{C}^{N} .
$$

We collect some further properties of this translation which will be used later on; for the proofs, the reader is referred to [28].

\section{Lemma 2.2.}

(1) For fixed $y \in \mathbb{R}^{N}, \tau_{y}$ is a continuous linear mapping from $C^{\infty}\left(\mathbb{R}^{N}\right)$ into $C^{\infty}\left(\mathbb{R}^{N}\right)$.

(2) For fixed $x, y \in \mathbb{R}^{N}$, the mapping $f \mapsto f\left(x *_{k} y\right)$ defines a compactly supported distribution. Its support is contained in the ball $\left\{\xi \in \mathbb{R}^{N}:|\xi| \leq|x|+|y|\right\}$.

(3) If $f \in \mathcal{S}\left(\mathbb{R}^{N}\right)$, then also $\tau_{y} f \in \mathcal{S}\left(\mathbb{R}^{N}\right)$, and $\left(\tau_{y} f\right)^{\wedge k}(\xi)=E_{k}(i y, \xi) \hat{f}^{k}(\xi)$. Moreover,

$$
\tau_{y} f(x)=\frac{1}{c_{k}} \int_{\mathbb{R}^{N}} \widehat{f}^{k}(\xi) E_{k}(i x, \xi) E_{k}(i y, \xi) w_{k}(\xi) d \xi
$$


(4) If $f, g \in \mathcal{S}\left(\mathbb{R}^{N}\right)$ and $x \in \mathbb{R}^{N}$, then

$$
\int_{\mathbb{R}^{N}} f\left(x *_{k} y\right) g(y) w_{k}(y) d y=\int_{\mathbb{R}^{N}} f(y) g\left(-x *_{k} y\right) w_{k}(y) d y .
$$

Remarks 2.3. Property (3) reveals that on $\mathcal{S}\left(\mathbb{R}^{N}\right)$, the translation (2.5) coincides with the version previously introduced in [22]. In [28, part (4) is shown only for compactly supported test functions; a simple density argument gives the result for Schwartz functions. Alternatively, (4) follows immediately from (3) and the Plancherel theorem for the Dunkl transform.

2.2. Expansion of $E_{k}$ in terms of $k$-spherical harmonics. In this section, we derive a series representation for the Dunkl kernel in terms of generalized spherical (" $k$-spherical") harmonics, which will be employed in the positivity proof for the Dunkl-type spherical mean operator. For a background in $k$-spherical harmonics, the reader may consult the recent monograph [10]. The expansion below is of some interest in its own and has been known for some time at least formally (a personal communication by M. de Jeu). In the $L^{2}$-sense, it can be readily deduced from the Dunkl-type Funk-Hecke formula (Theorem 5.3.4 of [10]). We do, however, give an alternative approach which yields better convergence and a special case of the Funk-Hecke formula as a corollary. Throughout this section it is assumed that $N \geq 2$. The space of $k$-spherical harmonics of degree $n \geq 0$ is defined by

$$
\mathcal{H}_{n}^{k}=\operatorname{ker} \Delta_{k} \cap \mathcal{P}_{n}^{N},
$$

where $\Delta_{k}$ is the Dunkl Laplacian and $\mathcal{P}_{n}^{N}$ denotes the space of homogeneous polynomials of degree $n$ on $\mathbb{R}^{N}$. The space $\mathcal{H}_{n}^{k}$ has a reproducing kernel $P_{n}^{k}(.,$.$) , which$ is defined by the property

$$
f(x)=d_{k}^{-1} \int_{S^{N-1}} f(y) P_{n}^{k}(x, y) w_{k}(y) d \sigma(y) \quad \text { for all } f \in \mathcal{H}_{n}^{k} \quad \text { and }|x|<1 .
$$

Here $S^{N-1}=\left\{x \in \mathbb{R}^{N}:|x|=1\right\}$ is the unit sphere in $\mathbb{R}^{N}, d \sigma$ denotes the Lebesgue surface measure and

$$
d_{k}=\int_{S^{N-1}} w_{k}(x) d \sigma(x)=\frac{c_{k}}{2^{\lambda} \Gamma(\lambda+1)},
$$

with

$$
\lambda=\gamma+N / 2-1 \geq 0 .
$$

Suppose that $\left\{Y_{n, j}: j=1, \ldots, d(n, N)\right\}$ is a real-coefficient orthonormal basis of $\mathcal{H}_{n}^{k}$ in $L^{2}\left(S^{N-1}, d_{k}^{-1} w_{k} d \sigma\right)$. In terms of this basis, $P_{n}^{k}$ is given by

$$
P_{n}^{k}(x, y)=\sum_{j=1}^{d(n, N)} Y_{n, j}(x) Y_{n, j}(y) .
$$

If $x, y \in S^{N-1}$, then according to Theorem 3.2 of [30], the kernel $P_{n}^{k}(x, y)$ can be written as

$$
P_{n}^{k}(x, y)=\frac{(n+\lambda)(2 \lambda)_{n}}{\lambda \cdot n !} V_{k} \widetilde{C}_{n}^{\lambda}(\langle x, .\rangle)(y)
$$


where the $\widetilde{C}_{n}^{\lambda}\left(n \in \mathbb{Z}_{+}\right)$are the (renormalized) Gegenbauer polynomials

$$
\begin{aligned}
\widetilde{C}_{n}^{\lambda}(x) & =\frac{(-1)^{n}}{2^{n}(\lambda+1 / 2)_{n}}\left(1-x^{2}\right)^{1 / 2-\lambda} \frac{d^{n}}{d x^{n}}\left(1-x^{2}\right)^{n+\lambda-1 / 2} \\
& ={ }_{2} F_{1}\left(-n, n+2 \lambda, \lambda+1 / 2 ; \frac{1-x}{2}\right) .
\end{aligned}
$$

Notice that in terms of this normalization, formula (2.7) remains true in the limiting case $\lambda=0$.

Proposition 2.4. Let $N \geq 2$. Then for all $x, y \in \mathbb{R}^{N}$ the Dunkl kernel $E_{k}(i x, y)$ admits the representation

$$
E_{k}(i x, y)=\sum_{n=0}^{\infty} \frac{\Gamma(\lambda+1)}{2^{n} \Gamma(n+\lambda+1)} j_{n+\lambda}(|x||y|) P_{n}^{k}(i x, y),
$$

where for $\alpha \geq-1 / 2, j_{\alpha}$ is the normalized spherical Bessel function

$$
j_{\alpha}(z)=\Gamma(\alpha+1) \cdot \sum_{n=0}^{\infty} \frac{(-1)^{n}(z / 2)^{2 n}}{n ! \Gamma(n+\alpha+1)} .
$$

The convergence of the series (2.9) is uniform on compact subsets of $\mathbb{R}^{N} \times \mathbb{R}^{N}$.

Proof. By Gegenbauer's degenerate form of the addition theorem for Bessel functions ([29], p. 368), we have

$$
e^{i r t}=\sum_{n=0}^{\infty}\left(\frac{i r}{2}\right)^{n} \frac{(2 \lambda)_{n}}{(\lambda)_{n} n !} j_{n+\lambda}(r) \widetilde{C}_{n}^{\lambda}(t) \quad \text { for all } r \in \mathbb{R}, t \in[-1,1]
$$

(with the obvious extension to the case $\lambda=0$ ). The series converges uniformly on every compact subset of $\mathbb{R} \times[-1,1]$. This is easily seen from the asymptotic behavior of the gamma function and the estimates

$$
\left|j_{n+\lambda}(r)\right| \leq 1, \quad\left|\widetilde{C}_{n}^{\lambda}(t)\right| \leq \widetilde{C}_{n}^{\lambda}(1)=1,
$$

which hold within the relevant ranges of $r$ and $t$. We now put $t=\langle x, y\rangle$ with $x, y \in S^{N-1}$, and apply the intertwining operator $V_{k}$ to both sides of (2.11). This may be done termwise, the locally uniform convergence being maintained: in fact, according to (2.3) together with (2.11) and (2.7), we obtain

$$
\begin{aligned}
E_{k}(i r x, y) & =V_{k}\left(e^{i r\langle x, .\rangle}\right)(y)=\int_{|\eta| \leq 1} e^{i r\langle x, \eta\rangle} d \mu_{y}^{k}(\eta) \\
& =\sum_{n=0}^{\infty} \frac{(2 \lambda)_{n}}{(\lambda)_{n} n !}\left(\frac{i r}{2}\right)^{n} j_{n+\lambda}(r) V_{k} \widetilde{C}_{n}^{\lambda}(\langle x, .\rangle)(y) \\
& =\sum_{n=0}^{\infty} \frac{\Gamma(\lambda+1)}{2^{n} \Gamma(n+\lambda+1)} j_{n+\lambda}(r) P_{n}^{k}(i r x, y) .
\end{aligned}
$$

Since $\left|V_{k} \widetilde{C}_{n}^{\lambda}(\langle x,\rangle).(y)\right| \leq \widetilde{C}_{n}^{\lambda}(1)=1$ for all $x, y \in S^{N-1}$, the series converges uniformly on compact subsets of $\mathbb{R} \times S^{N-1} \times S^{N-1}$. This implies the assertion.

In view of the orthogonality of the $k$-spherical harmonics $Y_{n, j}$, termwise spherical integration of (2.9) leads to the following well known special case of the Dunkl-type Funk-Hecke formula (Theorem 5.3.4 of [10]): 
Corollary 2.5. Let $N \geq 2$. Then for all $x \in \mathbb{R}^{N}$,

$$
\frac{1}{d_{k}} \int_{S^{N-1}} E_{k}(i x, y) Y_{n, j}(y) w_{k}(y) d \sigma(y)=\frac{\Gamma(\lambda+1)}{2^{n} \Gamma(n+\lambda+1)} j_{n+\lambda}(|x|) Y_{n, j}(i x) .
$$

2.3. The rank-one case. Here the reflection group is $G=\{i d, \sigma\}$, acting on $\mathbb{R}$ via $\sigma(x)=-x$. The corresponding kernel $E_{k}$ with parameter $k \geq 0$ has been calculated in 9 . It is given by

$$
E_{k}(z, w)=j_{k-1 / 2}(i z w)+\frac{z w}{2 k+1} j_{k+1 / 2}(i z w) ;
$$

thus $J_{k}(z, w)=j_{k-1 / 2}(i z w)$. It is well known (see e.g. [5], 3.5.61) that the Bessel functions $j_{\alpha}$ with $\alpha \geq-1 / 2$ satisfy a product formula of the form

$$
j_{\alpha}(x z) j_{\alpha}(y z)=\int_{0}^{\infty} j_{\alpha}(\xi z) d \nu_{x, y}^{\alpha}(\xi) \quad \text { for all } z \in \mathbb{C} .
$$

Here the $\nu_{x, y}^{\alpha}$ are probability measures on $\mathbb{R}_{+}$. For $x, y>0$, they are given by

$$
d \nu_{x, y}^{\alpha}(z)=m_{\alpha}(x, y, z) z^{2 \alpha+1} d z
$$

with the kernel

$m_{\alpha}(x, y, z)=\frac{2^{1-2 \alpha} \Gamma(\alpha+1)}{\sqrt{\pi} \Gamma\left(\alpha+\frac{1}{2}\right)} \cdot \frac{\left[\left(z^{2}-(x-y)^{2}\right)\left((x+y)^{2}-z^{2}\right)\right]^{\alpha-1 / 2}}{(x y z)^{2 \alpha}} \cdot 1_{[|x-y|, x+y]}(z)$.

The product formula (2.12) induces a convolution of point measures on $\mathbb{R}_{+}$according to

$$
\delta_{x} \circ_{\alpha} \delta_{y}:=\nu_{x, y}^{\alpha},
$$

which in turn allows a unique bilinear and weakly continuous extension to a probability-preserving convolution on $M_{b}\left(\mathbb{R}_{+}\right)$. It induces the structure of a commutative hypergroup on $\mathbb{R}_{+}$, called the Bessel-Kingman hypergroup of index $\alpha$, cf. [5]. The Dunkl kernel $E_{k}$ itself satisfies a product formula of type (1.4); see [21] and also [25]. Here the representing measures $\mu_{x, y}^{k}$ are not positive, but signed Borel measures on $\mathbb{R}$ with uniformly bounded total variation norm. For $x, y \neq 0$, one has

$$
d \mu_{x, y}^{k}(z)=m_{k-1 / 2}(|x|,|y|,|z|)|z|^{2 k} \cdot \frac{1-\sigma_{x, y, z}+\sigma_{z, x, y}+\sigma_{z, y, x}}{2} d z,
$$

with

$$
\sigma_{z, x, t}= \begin{cases}\frac{z^{2}+x^{2}-t^{2}}{2 z x} & \text { if } z, x \neq 0, \\ 0 & \text { else. }\end{cases}
$$

There exists a unique bilinear and separately weakly continuous convolution $*_{k}$ on $M_{b}(\mathbb{R})$ such that the convolution of point measures satisfies $\delta_{x} *_{k} \delta_{y}=\mu_{x, y}^{k}$. This convolution makes $M_{b}(\mathbb{R})$ into a commutative Banach $*$-algebra with unit $\delta_{0}$, involution $\mu^{*}(A)=\overline{\mu(-A)}$ and norm $\|\mu\|^{\prime}=\left\|L_{\mu}\right\|$, the operator $L_{\mu}$ on $M_{b}(\mathbb{R})$ being defined by $L_{\mu}(\nu)=\mu *_{k} \nu$. The Gelfand transform on $M_{b}(\mathbb{R})$ coincides with the corresponding Dunkl transform. For details, see [21]. Finally, the convolution $*_{k}$ matches the Dunkl-type generalized translation as defined above. In fact, Lemma 2.2 shows that $\int_{\mathbb{R}} f d\left(\delta_{x} *_{k} \delta_{y}\right)=f\left(x *_{k} y\right)$ for all $f \in \mathcal{S}(\mathbb{R})$, and hence, by a simple density argument, also for all $f \in C^{\infty}(\mathbb{R})$. 


\section{Positivity of the spherical mean operator}

Following [19] we define the Dunkl-type spherical mean operator $f \mapsto M_{f}$ on $C^{\infty}\left(\mathbb{R}^{N}\right)$ by

$$
M_{f}(x, t):=\frac{1}{d_{k}} \int_{S^{N-1}} f\left(x *_{k} t y\right) w_{k}(y) d \sigma(y) \quad\left(x \in \mathbb{R}^{N}, t \geq 0\right) .
$$

In case $N=1$ this reduces to

$$
M_{f}(x, t)=\frac{1}{2}\left(f\left(x *_{k} t\right)+f\left(x *_{k}-t\right)\right) .
$$

Lemma 2.2 easily implies that $M_{f} \in C^{\infty}\left(\mathbb{R}^{N} \times \mathbb{R}_{+}\right)$. The following is a key result of this paper:

Theorem 3.1. The spherical mean operator $f \mapsto M_{f}$ is positivity-preserving on $C^{\infty}\left(\mathbb{R}^{N}\right)$, i.e.,

$$
f \geq 0 \text { on } \mathbb{R}^{N} \quad \text { implies that } M_{f} \geq 0 \text { on } \mathbb{R}^{N} \times \mathbb{R}_{+} .
$$

Remark 3.2. The assertion is obvious in the rank-one case: In fact, $M_{f}$ can then be calculated explicitly in terms of the data given in Section[2.3. We have

$$
M_{f}(x, t)=\int_{\mathbb{R}} f(z) d \sigma_{x, t}^{k}(z)
$$

with

$$
\sigma_{x, t}^{k}=\frac{1}{2}\left(\mu_{x, t}^{k}+\mu_{x,-t}^{k}\right)
$$

which is easily checked to be a probability measure.

The proof of Theorem 3.1 in the higher rank case is achieved by a reduction to initial data of the form $f(x)=\Gamma_{k}(s, x, y)$, where $\Gamma_{k}$ is the Dunkl-type heat kernel

$$
\Gamma_{k}(s, x, y)=\frac{1}{(2 s)^{\gamma+N / 2} c_{k}} e^{-\left(|x|^{2}+|y|^{2}\right) / 4 s} E_{k}\left(\frac{x}{\sqrt{2 s}}, \frac{y}{\sqrt{2 s}}\right)
$$

$\left(x, y \in \mathbb{R}^{N}, s>0\right)$, see $\left[22\right.$. (Notice that the constant $c_{k}$ was defined differently there.) We recall from [22] that $\Gamma_{k}$ is strictly positive with

$$
\int_{\mathbb{R}^{N}} \Gamma_{k}(s, x, y) w_{k}(y) d y=1
$$

Moreover,

$$
\Gamma_{k}(s, x, y)=\frac{1}{c_{k}^{2}} \int_{\mathbb{R}^{N}} e^{-s|\xi|^{2}} E_{k}(-i x, \xi) E_{k}(i y, \xi) w_{k}(\xi) d \xi
$$

\section{Lemma 3.3.}

(1) For all $x, y, z \in \mathbb{R}^{N}$ and $s>0$,

$$
\Gamma_{k}\left(s, x *_{k} y, z\right)=\frac{1}{c_{k}^{2}} \int_{\mathbb{R}^{N}} e^{-s|\xi|^{2}} E_{k}(-i z, \xi) E_{k}(i x, \xi) E_{k}(i y, \xi) w_{k}(\xi) d \xi .
$$

In particular, $\Gamma_{k}\left(s, x *_{k} y, z\right)$ belongs to $\mathcal{S}\left(\mathbb{R}^{N}\right)$ as a function of each of the arguments $x, y, z$.

(2) $\int_{\mathbb{R}^{N}} \Gamma_{k}\left(s, x *_{k} y, z\right) w_{k}(z) d z=1$

(3) If $f \in \mathcal{S}\left(\mathbb{R}^{N}\right)$ and $(x, t) \in \mathbb{R}^{N} \times \mathbb{R}_{+}$, then

$$
M_{f}(x, t)=\lim _{s \downarrow 0} \int_{\mathbb{R}^{N}} M_{\Gamma_{k}(s, ., z)}(x, t) f(z) w_{k}(z) d z .
$$


Proof. (1) By the inversion theorem for the Dunkl transform, formula (3.2) is equivalent to

$$
\Gamma_{k}(s, ., z)^{\wedge k}(\xi)=c_{k}^{-1} e^{-s|\xi|^{2}} E_{k}(-i z, \xi) .
$$

Lemma 2.2(3) thus implies the stated identity. The rest follows from the invariance of $\mathcal{S}\left(\mathbb{R}^{N}\right)$ under the Dunkl transform.

(2) In view of (1), we write

$$
\Gamma_{k}\left(s, x *_{k} y, z\right)={\widehat{g_{x, y}}}^{k}(z), \quad \text { with } g_{x, y}(\xi):=\frac{1}{c_{k}} e^{-s|\xi|^{2}} E_{k}(i x, \xi) E_{k}(i y, \xi) .
$$

This gives

$$
\int_{\mathbb{R}^{N}} \Gamma_{k}\left(s, x *_{k} y, z\right) w_{k}(z) d z=\int_{\mathbb{R}^{N}}{\widehat{g_{x, y}}}^{k}(z) w_{k}(z) d z=c_{k} g_{x, y}(0)=1 .
$$

(3) By (1) and the definition of the Dunkl transform, one obtains

$$
\begin{aligned}
& \int_{\mathbb{R}^{N}} M_{\Gamma_{k}(s, ., z)}(x, t) f(z) w_{k}(z) d z \\
& \quad=\frac{1}{d_{k}} \int_{\mathbb{R}^{N}}\left(\int_{S^{N-1}} \Gamma_{k}\left(s, x *_{k} t y, z\right)(x, r) w_{k}(y) d \sigma(y)\right) f(z) w_{k}(z) d z \\
& =\frac{1}{c_{k} d_{k}} \int_{\mathbb{R}^{N}} \int_{S^{N-1}} e^{-s|\xi|^{2} \widehat{f}^{k}}(\xi) E_{k}(i x, \xi) E_{k}(i t y, \xi) w_{k}(y) d \sigma(y) w_{k}(\xi) d \xi .
\end{aligned}
$$

Thus, by the dominated convergence theorem,

$$
\begin{aligned}
\lim _{s \downarrow 0} \int_{\mathbb{R}^{N}} M_{\Gamma_{k}(s, ., z)}(x, t) f(z) w_{k}(z) d z \\
\quad=\frac{1}{c_{k} d_{k}} \int_{\mathbb{R}^{N}} \int_{S^{N-1}} \widehat{f}^{k}(\xi) E_{k}(i x, \xi) E_{k}(i t y, \xi) w_{k}(y) d \sigma(y) w_{k}(\xi) d \xi \\
\quad=\frac{1}{d_{k}} \int_{S^{N-1}} f\left(x *_{k} t y\right) w_{k}(y) d \sigma(y)=M_{f}(x, t) .
\end{aligned}
$$

Remark 3.4. A similar calculation shows that for $f \in \mathcal{S}\left(\mathbb{R}^{N}\right)$ and $x, y \in \mathbb{R}^{N}$,

$$
f\left(x *_{k} y\right)=\lim _{s \downarrow 0} \int_{\mathbb{R}^{N}} f(z) \Gamma_{k}\left(s, x *_{k} y, z\right) w_{k}(z) d z .
$$

Proof of Theorem 3.1. We may assume that $N \geq 2$ and $\gamma>0$, hence also $\lambda>0$. Moreover, it suffices to prove the result for $f \in \mathcal{S}\left(\mathbb{R}^{N}\right)$, because $\mathcal{S}\left(\mathbb{R}^{N}\right)$ is dense in $C^{\infty}\left(\mathbb{R}^{N}\right)$ and $M_{f}(x, t)$ depends continuously on $f$ as a consequence of Lemma 2.2(2). Thus, by part (3) of Lemma 3.3, it remains to show that

$$
M_{\Gamma_{k}(s, ., z)}(x, t)>0 \text { for all } s \geq 0, z \in \mathbb{R}^{N} \text { and all } t \geq 0, x \in \mathbb{R}^{N} .
$$

Let us write $M(x, t):=M_{\Gamma_{k}(s, ., z)}(x, t)$ for brevity. Invoking part (1) of Lemma 3.3 and Corollary 2.5 leads to

$$
\begin{aligned}
M(x, t) & =\frac{1}{d_{k}} \int_{S^{N-1}} \Gamma_{k}\left(s, x *_{k} t y, z\right) w_{k}(y) d \sigma(y) \\
& =\frac{1}{c_{k}^{2}} \int_{\mathbb{R}^{N}} e^{-s|\xi|^{2}} E_{k}(-i z, \xi) E_{k}(i x, \xi) j_{\lambda}(t|\xi|) w_{k}(\xi) d \xi \\
& =\frac{d_{k}}{c_{k}^{2}} \int_{0}^{\infty} I(x, z, r) e^{-s r^{2}} j_{\lambda}(t r) r^{2 \lambda+1} d r
\end{aligned}
$$


with

$$
I(x, z, r)=\frac{1}{d_{k}} \int_{S^{N-1}} E_{k}(i x, r \xi) E_{k}(-i z, r \xi) w_{k}(\xi) d \sigma(\xi) .
$$

This integral has to be brought into a form from which the positivity of $M(x, t)$ can be read off. For this, we insert the series expansion (2.9) for $E_{k}(i x, r \xi)$, thus obtaining

$$
\begin{aligned}
I(x, z, r)= & \frac{1}{d_{k}} \sum_{n=0}^{\infty} \frac{\Gamma(\lambda+1)}{2^{n} \Gamma(n+\lambda+1)} j_{n+\lambda}(r|x|) . \\
& \cdot \int_{S^{N-1}} P_{n}^{k}(i r x, \xi) E_{k}(-i r z, \xi) w_{k}(\xi) d \sigma(\xi) \\
= & \sum_{n=0}^{\infty}\left(\frac{\Gamma(\lambda+1)}{2^{n} \Gamma(n+\lambda+1)}\right)^{2} j_{n+\lambda}(r|x|) j_{n+\lambda}(r|z|) P_{n}^{k}(i r x,-i r z),
\end{aligned}
$$

where for the second identity, again Corollary 2.5 was used. In case $x, z \neq 0$, the homogeneity of $P_{n}^{k}$ allows one to write

$$
P_{n}^{k}(i r x,-i r z)=\frac{(n+\lambda)(2 \lambda)_{n}}{\lambda \cdot n !}\left(r^{2}|x||z|\right)^{n} V_{k} \widetilde{C}_{n}^{\lambda}\left(\left\langle\frac{x}{|x|}, .\right\rangle\right)\left(\frac{z}{|z|}\right) .
$$

We now employ a well-known degenerate version of the addition theorem for Gegenbauer polynomials (see [2], (4.36)): For all $s, t, \theta \in \mathbb{R}$,

$$
\begin{aligned}
& j_{\lambda}\left(\sqrt{s^{2}+t^{2}-2 s t \cos \theta}\right) \\
& \quad=\sum_{n=0}^{\infty} \frac{(n+\lambda)(2 \lambda)_{n}}{\lambda \cdot n !}\left(\frac{\Gamma(\lambda+1)}{2^{n} \Gamma(n+\lambda+1)}\right)^{2}(s t)^{n} j_{n+\lambda}(s) j_{n+\lambda}(t) \widetilde{C}_{n}^{\lambda}(\cos \theta) .
\end{aligned}
$$

This series converges uniformly with respect to $\theta \in \mathbb{R}$. Combining (3.6) and (3.7) and recalling the Laplace representation (2.2) for $V_{k}$, we now see that for all $x, z \neq 0$,

$$
\begin{aligned}
I(x, z, r) & =\int_{\mathbb{R}^{N}} j_{\lambda}\left(r \sqrt{|x|^{2}+|z|^{2}-2|z|\langle x, \eta\rangle}\right) d \mu_{z /|z|}^{k}(\eta) \\
& =\int_{\mathbb{R}^{N}} j_{\lambda}\left(r \sqrt{|x|^{2}+|z|^{2}-2\langle x, \eta\rangle}\right) d \mu_{z}^{k}(\eta) .
\end{aligned}
$$

The second identity follows from the dilation equivariance of $\mu_{z}^{k}: \mu_{r z}^{k}(A)=\mu_{z}^{k}\left(r^{-1} A\right)$ for all $r>0, A \in \mathcal{B}\left(\mathbb{R}^{N}\right)$, cf. [23]. By virtue of Corollary [2.5] (3.8) remains true if $x=0$ or $z=0$. For abbreviation, put

$$
v_{z}(\eta):=\sqrt{|x|^{2}+|z|^{2}-2\langle x, \eta\rangle} .
$$

Then by (3.4), the product formula (2.12) for the Bessel functions $j_{\lambda}$ and 11.4.29 of [1, we arrive at

$$
\begin{aligned}
M(x, t) & =\frac{d_{k}}{c_{k}^{2}} \int_{\mathbb{R}^{N}} \int_{0}^{\infty} j_{\lambda}\left(r v_{z}(\eta)\right) j_{\lambda}(r t) e^{-s r^{2}} r^{2 \lambda+1} d r d \mu_{z}^{k}(\eta) \\
& =\frac{d_{k}}{c_{k}^{2}} \int_{\mathbb{R}^{N}} \int_{0}^{\infty}\left(\int_{0}^{\infty} j_{\lambda}(r u) e^{-s r^{2}} r^{2 \lambda+1} d r\right) d \nu_{v_{z}(\eta), t}^{\lambda}(u) d \mu_{z}^{k}(\eta) \\
& =\frac{d_{k}}{c_{k}^{2}} \frac{\Gamma(\lambda+1)}{2 s^{\lambda+1}} \int_{\mathbb{R}^{N}}\left(\int_{0}^{\infty} e^{-u^{2} / 4 s} d \nu_{v_{z}(\eta), t}^{\lambda}(u)\right) d \mu_{z}^{k}(\eta),
\end{aligned}
$$

which is obviously nonnegative. This finishes the proof. 


\section{A positive Radial product formula for the DUNKL KERnel}

4.1. Statement of the main result. Consider $f(x)=E_{k}(i x, z)$ with $z \in \mathbb{R}^{N}$. Then

$$
M_{f}(x, t)=E_{k}(i x, z) j_{\lambda}(t|z|) .
$$

Indeed, we have

$$
\frac{1}{d_{k}} \int_{S^{N-1}} E_{k}(i x, z) w_{k}(z) d \sigma(z)=j_{\lambda}(|x|)
$$

which follows from Corollary 2.5 if $N \geq 2$ and is obviously also true if $N=1$. In view of (2.6), this gives (4.1). Theorem 3.1 easily implies the existence of the representing measures stated in the next theorem, which is the main result of this paper.

Theorem 4.1. For each $x \in \mathbb{R}^{N}$ and $t \in \mathbb{R}_{+}$there exists a unique compactly supported probability measure $\sigma_{x, t}^{k} \in M^{1}\left(\mathbb{R}^{N}\right)$ such that

$$
E_{k}(i x, y) j_{\lambda}(t|y|)=\int_{\mathbb{R}^{N}} E_{k}(i \xi, y) d \sigma_{x, t}^{k}(\xi) \quad \text { for all } y \in \mathbb{R}^{N}
$$

It represents the spherical mean operator $f \mapsto M_{f}$ in the sense that

$$
M_{f}(x, t)=\int_{\mathbb{R}^{N}} f d \sigma_{x, t}^{k} \quad \text { for all } f \in C^{\infty}\left(\mathbb{R}^{N}\right) .
$$

The measure $\sigma_{x, t}^{k}$ satisfies

$$
\operatorname{supp} \sigma_{x, t}^{k} \subseteq \bigcup_{g \in G}\left\{\xi \in \mathbb{R}^{N}:|\xi-g x| \leq t\right\},
$$

and the mapping $(x, t) \mapsto \sigma_{x, t}^{k}$ is continuous with respect to the weak topology on $M^{1}\left(\mathbb{R}^{N}\right)$. Moreover,

$$
\sigma_{g x, t}^{k}(A)=\sigma_{x, t}^{k}\left(g^{-1}(A)\right) \quad \text { and } \quad \sigma_{r x, r t}^{k}(A)=\sigma_{x, t}^{k}\left(r^{-1} A\right)
$$

for all $g \in G, r>0$, and all Borel sets $A \in \mathcal{B}\left(\mathbb{R}^{N}\right)$.

Remarks 4.2 .

1. The above result also gives a natural extension of the spherical mean operator at hand: namely, for $f \in C_{b}\left(\mathbb{R}^{N}\right)$, we may define $M_{f} \in C_{b}\left(\mathbb{R}^{N} \times \mathbb{R}_{+}\right)$by

$$
M_{f}(x, t):=\int_{\mathbb{R}^{N}} f d \sigma_{x, t}^{k} .
$$

2. In the rank-one case, the support of $\sigma_{x, t}^{k}$ with $t \neq 0$ is given by

$$
\operatorname{supp} \sigma_{x, t}^{k}=[-|x|-t,-|| x|-t|] \cup[|| x|-t|,|x|+t] .
$$

This illustrates that the complete $G$-orbit of $x$ has to be taken into account in the description of $\operatorname{supp} \sigma_{x, t}^{k}$.

3. From part (2) of Lemma 2.2, a weaker statement on the support of $\sigma_{x, t}^{k}$ is immediate, namely $\operatorname{supp} \sigma_{x, t}^{k} \subseteq\left\{\xi \in \mathbb{R}^{N}:|\xi| \leq|x|+t\right\}$.

In order to derive the stated properties of the support of $\sigma_{x, t}^{k}$ we use an approach via a Darboux-type initial value problem satisfied by the spherical mean $M_{f}$. For $\alpha \geq-1 / 2$, denote by $A_{\alpha}$ the singular Sturm-Liouville operator

$$
A_{\alpha}:=\partial_{t}^{2}+\frac{2 \alpha+1}{t} \partial_{t}
$$


on $\mathbb{R}_{+}$. The Bessel functions $t \mapsto j_{\alpha}(t z), z \in \mathbb{C}$, are (up to normalization) the unique even and analytic eigenfunctions of this operator, satisfying

$$
A_{\alpha}^{t} j_{\alpha}(t z)=-z^{2} j_{\alpha}(t z)
$$

here the upper index indicates the relevant variable.

For $f \in \mathcal{S}\left(\mathbb{R}^{N}\right)$, we may write

$$
M_{f}(x, t)=\frac{1}{c_{k}} \int_{\mathbb{R}^{N}} \widehat{f}^{k}(\xi) E_{k}(x, i \xi) j_{\lambda}(t|\xi|) w_{k}(\xi) d \xi .
$$

In this case, a direct calculation verifies that $u=M_{f}$ solves the following initial value problem for the Darboux-type differential-reflection operator $\Delta_{k}^{x}-A_{\lambda}^{t}$ :

$$
\begin{aligned}
& \left(\Delta_{k}^{x}-A_{\lambda}^{t}\right) u=0 \quad \text { in } \mathbb{R}^{N} \times \mathbb{R}_{+} ; \\
& u(x, 0)=f(x), u_{t}(x, 0)=0 \text { for all } x \in \mathbb{R}^{N} .
\end{aligned}
$$

(To obtain the initial data, one has to use the inversion theorem for the Dunkl transform as well as the fact that $M_{f}$ is even with respect to $t$.) In [19], Prop. 5.2, it is shown that for arbitrary $f \in C^{\infty}\left(\mathbb{R}^{N}\right), M_{f}$ is in fact the unique $C^{\infty}$-solution of (4.5). Instead of studying the domain of dependence for the above Darbouxtype operator directly, we shall consider the corresponding reflection-invariant wave operator, which is easier to handle.

4.2. Domain of dependence for wave operators related to reflection groups. This section is devoted to the study of the Dunkl-type wave operator $L_{k}-\partial_{t}^{2}$, where $L_{k}$ denotes the reflection-invariant part of the Dunkl Laplacian $\Delta_{k}$, cf. (2.1). Notice that in the rank-one case, $L_{k}$ coincides with the Sturm-Liouville operator $A_{k-1 / 2}$. It will be important for the following that $L_{k}$ can be written in divergence form,

$$
L_{k}=\frac{1}{w_{k}(x)} \sum_{i=1}^{N} \partial_{x_{i}}\left(w_{k}(x) \partial_{x_{i}}\right) .
$$

Let us now turn to the Dunkl-type wave equation on $\mathbb{R}^{N} \times \mathbb{R}_{+}$associated with $G$ and $k$,

$$
\left(L_{k}-\partial_{t}^{2}\right) u=0 .
$$

The following uniqueness result for solutions of this equation is in close analogy to well-known facts in the classical case $k=0$.

Lemma 4.3. Suppose that $u$ is a real-valued $C^{2}$-solution of (4.7), given in the truncated cone

$$
C\left(x_{0}, t_{0}\right)=\left\{(x, t) \in \mathbb{R}^{N} \times \mathbb{R}_{+}:\left|x-x_{0}\right| \leq t_{0}-t\right\} .
$$

Define the energy of $u$ at time $t$ within this cone by

$$
E(t):=\frac{1}{2} \int_{B_{t}}\left(u_{t}^{2}+\left|\nabla_{x} u(x, t)\right|^{2}\right) w_{k}(x) d x,
$$

where the integration is taken over the level set

$$
B_{t}=\left\{x \in \mathbb{R}^{N}:\left|x-x_{0}\right| \leq t_{0}-t\right\} .
$$

Then $E^{\prime} \leq 0$ on $\mathbb{R}_{+}$. 
Proof. The chain rule gives

$$
E^{\prime}(t)=I(t)-\frac{1}{2} \int_{\partial B_{t}}\left(u_{t}(x, t)^{2}+\left|\nabla_{x} u(x, t)\right|^{2}\right) w_{k}(x) d x
$$

with

$$
\begin{aligned}
I(t)= & \int_{B_{t}}\left(u_{t}(x, t) u_{t t}(x, t)+\sum_{j} u_{x_{j}}(x, t) u_{t, x_{j}}(x, t)\right) w_{k}(x) d x \\
= & \int_{B_{t}}\left(u_{t t}(x, t)-L_{k}^{x} u(x, t)\right) u_{t}(x, t) w_{k}(x) d x \\
& \quad+\int_{B_{t}} \sum_{j=1}^{N}\left(u_{t}(x, t) u_{x_{j}}(x, t) w_{k}(x)\right)_{x_{j}} d x \\
= & \int_{\partial B_{t}} u_{t}(x, t) \partial_{\nu} u(x, t) w_{k}(x) d \sigma(x),
\end{aligned}
$$

where the second identity follows from (4.6) and the last one from the divergence theorem ( $\nu$ is the exterior unit normal to $B_{t}$ and $d \sigma$ the Lebesgue surface measure on $\left.\partial B_{t}\right)$. By the Cauchy-Schwarz inequality,

$$
u_{t} \partial_{\nu} u \leq \frac{1}{2}\left(u_{t}^{2}+\left|\nabla_{x} u\right|^{2}\right) .
$$

This implies the assertion.

The following uniqueness result is an immediate consequence:

Theorem 4.4. Suppose that $u$ is a $C^{2}$-solution of the wave equation $\left(L_{k}-\partial_{t}^{2}\right) u=0$, defined in the cone $C\left(x_{0}, t_{0}\right)$ and satisfying

$$
u_{t}(x, 0)=u(x, 0)=0 \quad \text { for all } x \in \mathbb{R}^{N} \text { with }\left|x-x_{0}\right| \leq t_{0} .
$$

Then $u$ vanishes in $C\left(x_{0}, t_{0}\right)$.

Proof. We may assume that $u$ is real-valued. Since $E(t) \geq 0$ and $E(0)=0$, the lemma shows that $E(t)=0$ for $0 \leq t \leq t_{0}$, and hence $u_{t}=0$ and $\nabla_{x} u=0$ in $C\left(x_{0}, t_{0}\right)$. This implies the assertion.

We mention that there exists a thorough study of wave operators (and more general hyperbolic operators) related to root systems especially in the case of integervalued multiplicity functions; see [3] and the references cited there. In particular, for integer-valued $k$ and odd dimensions $N$ satisfying $N \geq 2 \gamma+3$, the wave operator $L_{k}-\partial_{t}^{2}$ in fact satisfies the strong Huygens principle. This means that a solution at $\left(x_{0}, t_{0}\right)$ depends only on the data in an infinitesimal neighborhood of the surface of the propagation cone with vertex at $\left(x_{0}, t_{0}\right)$.

4.3. Proof of the main result. For the proof of the properties of the representing measures in Theorem 4.1 we have to relate the Darboux-type equation (4.5) to the hyperbolic equation (4.7). For this, we involve the Riemann-Liouville transform with parameter $\alpha>-1 / 2$ on $\mathbb{R}_{+}$. It is given by

$$
\mathcal{R}_{\alpha} f(t)=\frac{2 \Gamma(\alpha+1)}{\Gamma(1 / 2) \Gamma(\alpha+1 / 2)} \int_{0}^{1} f(s t)\left(1-s^{2}\right)^{\alpha-1 / 2} d s
$$


for $f \in C^{\infty}\left(\mathbb{R}_{+}\right)$, see [27]. The operator $\mathcal{R}_{\alpha}$ satisfies the intertwining property

$$
A_{\alpha} \mathcal{R}_{\alpha}=\mathcal{R}_{\alpha} \frac{d^{2}}{d t^{2}}
$$

Notice that the rank-one intertwining operator $V_{k}$, when restricted to even functions, just coincides with $\mathcal{R}_{k-1 / 2}$. According to [27, $\mathcal{R}_{\alpha}$ is a topological isomorphism of $C^{\infty}\left(\mathbb{R}_{+}\right)$with respect to the usual Fréchet topology, which is induced from the identification of $C^{\infty}\left(\mathbb{R}_{+}\right)$with $\left\{f \in C^{\infty}(\mathbb{R}): f(-t)=f(t)\right\}$.

Proof of Theorem 4.1. For fixed $x \in \mathbb{R}^{N}$ and $t \geq 0$, consider the linear functional

$$
\Phi_{x, t}: f \mapsto M_{f}(x, t),
$$

which is positivity-preserving on $C^{\infty}\left(\mathbb{R}^{N}\right)$ according to Theorem 3.1. Moreover, $\Phi_{x, t}(1)=1$. It follows that $\Phi_{x, t}$ is represented by a compactly supported probability measure $\sigma_{x, t}^{k} \in M^{1}\left(\mathbb{R}^{N}\right.$ ) (cf. Theorem 2.1.7 of [13]). This implies statement (4.3) and also (4.2) in view of relation 4.1). Notice that

$$
E_{k}(i x, z) j_{\lambda}(t|z|)=\left(\sigma_{x, t}^{k}\right)^{\wedge k}(-z) .
$$

Thus the uniqueness of $\sigma_{x, t}^{k}$ follows from the injectivity of the Dunkl transform on $M^{1}\left(\mathbb{R}^{N}\right)$, cf. Proposition 2.1 In order to check the weak continuity of $(x, t) \mapsto \sigma_{x, t}^{k}$, take a sequence $\left(x_{n}, t_{n}\right)_{n \in \mathbb{N}} \subset \mathbb{R}^{N} \times \mathbb{R}_{+}$with $\lim _{n \rightarrow \infty}\left(x_{n}, t_{n}\right)=\left(x_{0}, t_{0}\right)$. Then (4.9) implies that $\left(\sigma_{x_{n}, t_{n}}^{k}\right)^{\wedge k} \rightarrow\left(\sigma_{x_{n}, t_{n}}^{k}\right)^{\wedge k}$ pointwise on $\mathbb{R}^{N}$. Lévy's continuity theorem for the Dunkl transform (Lemma 2.1(5)) now yields that the $\sigma_{x_{n}, t_{n}}^{k}$ converge weakly to $\sigma_{x_{0}, t_{0}}^{k}$. Further, the claimed transformation properties of $\sigma_{x, t}^{k}$ are immediate consequences of the invariance properties (2.4) of the kernel $E_{k}$. It remains to analyse the support of $\sigma_{x, t}^{k}$. For this, we shall employ Theorem 4.4. We therefore first reduce the general situation to the group-invariant case. We introduce the group means

$$
\widetilde{\sigma}_{x, t}^{k}:=\frac{1}{|G|} \sum_{g \in G} \sigma_{g x, t}^{k}=\frac{1}{|G|} \sum_{g \in G} \sigma_{x, t}^{k} \circ g^{-1} .
$$

Since $\sigma_{x, t}^{k}$ is positive, it is enough to show that $\widetilde{\sigma}_{x, t}^{k}$ is supported in

$$
K(x, t):=\bigcup_{g \in G}\left\{\xi \in \mathbb{R}^{N}:|\xi-g x| \leq t\right\},
$$

as stated for $\sigma_{x, t}^{k}$. For this in turn, it suffices to show that

$$
\int_{\mathbb{R}^{N}} f d \widetilde{\sigma}_{x, t}^{k}=0
$$

for all $G$-invariant $f \in \mathcal{S}\left(\mathbb{R}^{N}\right)$ whose support does not intersect $K(x, t)$. So suppose that $f \in \mathcal{S}\left(\mathbb{R}^{N}\right)$ is $G$-invariant. Then according to Proposition 2.4 of [24], $\widehat{f}^{k}$ is also $G$-invariant, and (4.4) shows that $x \mapsto M_{f}(x, t)$ is $G$-invariant as well. Put $u_{f}(x, t):=\left(\mathcal{R}_{\lambda}^{t}\right)^{-1} M_{f}(x, t)$, which is still $G$-invariant with respect to $x$. According to (4.5) and the intertwining property of the Riemann-Liouville transform, $u=u_{f}$ belongs to $C^{\infty}\left(\mathbb{R}^{N} \times \mathbb{R}_{+}\right)$and solves the initial value problem

$$
\begin{aligned}
& \left(L_{k}-\partial_{t}^{2}\right) u=0 \quad \text { in } \mathbb{R}^{N} \times \mathbb{R}_{+} ; \\
& u(x, 0)=f(x), u_{t}(x, 0)=0 \text { for all } x \in \mathbb{R}^{N} .
\end{aligned}
$$


Now suppose in addition that supp $f \cap K(x, t)=\emptyset$. Then Theorem4.4implies that $u_{f}(x, s)=0$ for all $0 \leq s \leq t$. From the explicit form (4.8) of the Riemann-Liouville transform $\mathcal{R}_{\lambda}$ we further deduce that

$$
\int_{\mathbb{R}^{N}} f d \widetilde{\sigma}_{x, t}^{k}=M_{f}(x, t)=\mathcal{R}_{\lambda}^{t} u_{f}(x, t)=0,
$$

as claimed. This completes the proof of Theorem 4.1.

\section{Positive translation of Radial Functions}

In this section, we derive a slightly weaker variant of Theorem 4.1 For its formulation, we introduce some additional notation: If $\mathcal{F}$ is a space of $\mathbb{C}$-valued functions on $\mathbb{R}^{N}$, denote by

$$
\mathcal{F}_{\text {rad }}:=\{f \in \mathcal{F}: f \circ A=f \text { for all } A \in O(N, \mathbb{R})\}
$$

the subspace of those $f \in \mathcal{F}$ that are radial. For $f \in \mathcal{F}_{\text {rad }}$ there exists a unique function $\widetilde{f}: \mathbb{R}_{+} \rightarrow \mathbb{C}$ such that $f(x)=\widetilde{f}(|x|)$ for all $x \in \mathbb{R}^{N}$. Similarly, if $\mathcal{M}$ is a space of Borel measures on $\mathbb{R}^{N}$, then

$$
\mathcal{M}_{\text {rad }}:=\{\mu \in \mathcal{M}: \mu \circ A=\mu \text { for all } A \in O(N, \mathbb{R})\}
$$

denotes the subspace of radial measures from $\mathcal{M}$.

Theorem 5.1. For each $x, y \in \mathbb{R}^{N}$ there exists a unique compactly supported, radial probability measure $\rho_{x, y}^{k} \in M_{\text {rad }}^{1}\left(\mathbb{R}^{N}\right)$ such that for all $f \in C_{\text {rad }}^{\infty}\left(\mathbb{R}^{N}\right)$,

$$
f\left(x *_{k} y\right)=\int_{\mathbb{R}^{N}} f d \rho_{x, y}^{k} .
$$

The support of $\rho_{x, y}^{k}$ is contained in

$$
\left\{\xi \in \mathbb{R}^{N}: \min _{g \in G}|x+g y| \leq|\xi| \leq \max _{g \in G}|x+g y|\right\} .
$$

In particular, if $0 \in \operatorname{supp} \rho_{x, y}^{k}$, then the $G$-orbits of $x$ and $-y$ coincide.

Proof. Take $f \in \mathcal{S}_{\text {rad }}\left(\mathbb{R}^{N}\right)$. Then according to Proposition 2.4 of [24], $\widehat{f}^{k} \in$ $\mathcal{S}_{\text {rad }}\left(\mathbb{R}^{N}\right)$ with $\widehat{f}^{k}(\xi)=\mathcal{H}^{\lambda}(\widetilde{f})(|\xi|)$. Here $\mathcal{H}^{\lambda}$ stands for the Hankel transform of index $\lambda$ on $L^{1}\left(\mathbb{R}_{+}, r^{2 \lambda+1} d r\right)$, given by

$$
\mathcal{H}^{\lambda}(g)(s)=\frac{1}{2^{\lambda} \Gamma(\lambda+1)} \int_{0}^{\infty} g(r) j_{\lambda}(r s) r^{2 \lambda+1} d r .
$$

Employing Lemma 2.2 (3), relations (3.5), (3.8) as well as the inversion theorem for the Hankel transform, one obtains

$$
\begin{aligned}
f( & \left.x *_{k} y\right)=\frac{1}{c_{k}} \int_{0}^{\infty} \mathcal{H}^{\lambda}(\widetilde{f})(r) \int_{S^{N-1}} E_{k}(i x, r \xi) E_{k}(i y, r \xi) w_{k}(\xi) d \sigma(\xi) r^{2 \lambda+1} d r \\
& =\frac{d_{k}}{c_{k}} \int_{0}^{\infty} \mathcal{H}^{\lambda}(\widetilde{f})(r) I(-x, y, r) r^{2 \lambda+1} d r \\
& =\frac{1}{2^{\lambda} \Gamma(\lambda+1)} \int_{\mathbb{R}^{N}} \int_{0}^{\infty} \mathcal{H}^{\lambda}(\widetilde{f})(r) j_{\lambda}\left(r \sqrt{|x|^{2}+|y|^{2}+2\langle x, \eta\rangle}\right) r^{2 \lambda+1} d r d \mu_{y}^{k}(\eta) \\
& =\int_{C(y)} \tilde{f}\left(\sqrt{|x|^{2}+|y|^{2}+2\langle x, \eta\rangle}\right) d \mu_{y}^{k}(\eta)
\end{aligned}
$$


here for the second identity, we used the fact that $I(x,-y, r)=I(-x, y, r)$. This shows that $f\left(x *_{k} y\right) \geq 0$ if $f \geq 0$ on $\mathbb{R}^{N}$. Since $\mu_{y}^{k}$ is a compactly supported probability measure, it also follows that

$$
\sup \left\{\left|f\left(x *_{k} y\right)\right|: f \in \mathcal{S}\left(\mathbb{R}^{N}\right),\|f\|_{\infty} \leq 1\right\}=1 .
$$

Similarly to the proof of Theorem 4.1 we proceed by considering the linear functional $\Psi_{x, y}: f \mapsto f\left(x *_{k} y\right)$, which is positive and bounded with norm $\left\|\Psi_{x, y}\right\|=1$ on the dense subspace $\mathcal{S}_{\text {rad }}\left(\mathbb{R}^{N}\right)$ of $\left(C_{0, \text { rad }}\left(\mathbb{R}^{N}\right),\|\cdot\|_{\infty}\right)$. Its continuous extension to $C_{0, r a d}\left(\mathbb{R}^{N}\right)$ is a probability measure $\rho_{x, y}^{k} \in M_{\text {rad }}^{1}\left(\mathbb{R}^{N}\right)$ that satisfies (5.1). Finally, the statement concerning the support of $\rho_{x, y}^{k}$ is immediate from the implication

$$
\eta \in C(y) \Longrightarrow \min _{g \in G}|x+g y| \leq \sqrt{|x|^{2}+|y|^{2}+2\langle x, \eta\rangle} \leq \max _{g \in G}|x+g y| .
$$

The information on the support of the measures $\rho_{x, y}^{k}$ can in turn be used to obtain a sharper statement on the supports of the representing measures for the spherical mean operator in Theorem 4.1:

Corollary 5.2. The measures $\sigma_{x, t}^{k}$ from Theorem 4.1 satisfy

$$
\operatorname{supp} \sigma_{x, t}^{k} \subseteq\left\{\xi \in \mathbb{R}^{N}:|\xi| \geq|| x|-t|\right\} .
$$

In particular, if $0 \in \operatorname{supp} \sigma_{x, t}^{k}$, then $|x|=t$.

Proof. Suppose to the contrary that $\operatorname{supp} \sigma_{x, t}^{k} \nsubseteq\left\{\xi \in \mathbb{R}^{N}:|\xi| \geq|| x|-t|\right\}$. Then there exists some $f \in \mathcal{S}_{\text {rad }}\left(\mathbb{R}^{N}\right)$ with $f \geq 0$,

$$
\operatorname{supp} f \cap\left\{\xi \in \mathbb{R}^{N}:|\xi| \geq|| x|-t|\right\}=\emptyset,
$$

and such that $M_{f}(x, t)>0$. But then $\eta \mapsto f\left(x *_{k} t \eta\right)$ is not identically zero on $S^{N-1}$. In view of Theorem [5.1, this is a contradiction to (5.2).

\section{An application: Semigroups of $k$-Invariant Markov Kernels WITH RADIAL DISTRIBUTIONS}

There is a concept of homogeneity for Markov processes on $\mathbb{R}^{N}$, called $k$-invariance, which generalizes the classical notion of processes with independent, stationary increments to the Dunkl setting. This was introduced and studied in some detail in 24. The most important example is a generalization of Brownian motion, with the transition probabilities given in terms of the generalized heat kernel $\Gamma_{k}$, cf. example 6.8 (1) below. A Dunkl-type Brownian motion is a Feller process and therefore admits a version with càdlàg paths. This version gives, after symmetrization with respect to the underlying reflection group, a diffusion on the Weyl chambers. $k$-invariant Markov processes are constructed from semigroups of Markov kernels that are $k$-invariant in the following sense:

Definition 6.1 (24]). A Markov kernel $P: \mathbb{R}^{N} \times \mathcal{B}\left(\mathbb{R}^{N}\right) \rightarrow[0,1]$ is called $k$ invariant if

$$
P(x, .)^{\wedge k}(\xi)=P(0, .)^{\wedge k}(\xi) \cdot E_{k}(-i x, \xi) \quad \text { for all } x, \xi \in \mathbb{R}^{N} .
$$

Here $P(x,$.$) is regarded as a probability measure on \mathbb{R}^{N}$. 
If $k=0$, the $k$-invariant Markov kernels are exactly those that are translationinvariant, which means that they satisfy $P\left(x+x_{0}, A+x_{0}\right)=P(x, A)$ for all $x, x_{0} \in$ $\mathbb{R}^{N}$ and all $A \in \mathcal{B}\left(\mathbb{R}^{N}\right)$. Equivalently, the translation-invariant Markov kernels (with $k=0$ ) are those of the form $P(x, A):=\delta_{x} * \mu(A)$ with a probability measure $\mu \in M^{1}\left(\mathbb{R}^{N}\right)$; here $*$ denotes the usual group convolution. If $k>0$, then for given $\mu \in M^{1}\left(\mathbb{R}^{N}\right)$ there usually exists no $k$-invariant Markov kernel such that $P(0,)=.\mu$. This is due to the fact that the associated generalized translation on $\mathbb{R}^{N}$ cannot be expected to be probability-preserving (and is definitely not in the rank-one case).

However, our results allow us to define associated $k$-invariant Markov kernels for all measures $\mu \in M^{1}\left(\mathbb{R}^{N}\right)$ belonging to the class

$$
M_{k}^{1}\left(\mathbb{R}^{N}\right):=\left\{\mu \in M^{1}\left(\mathbb{R}^{N}\right): w_{k}^{-1}(x) d \mu(x) \text { is radial }\right\} .
$$

This leads to a considerable variety of $k$-invariant Markov processes beyond those discussed in 24. Concerning the definition of $M_{k}^{1}\left(\mathbb{R}^{N}\right)$, one should notice that for $\mu \in M_{k}^{1}\left(\mathbb{R}^{N}\right)$, the measure $w_{k}^{-1} d \mu$ need not be a Radon measure.

Proposition 6.2. Let $\mu \in M_{k}^{1}\left(\mathbb{R}^{N}\right)$. Then for each $x \in \mathbb{R}^{N}$, there exists a unique probability measure $\delta_{x} *_{k} \mu \in M^{1}\left(\mathbb{R}^{N}\right)$ such that

$$
\left(\delta_{x} *_{k} \mu\right)(f)=\int_{\mathbb{R}^{N}} f\left(x *_{k} y\right) d \mu(y) \quad \text { for all } f \in \mathcal{S}\left(\mathbb{R}^{N}\right) .
$$

It satisfies

$$
\left(\delta_{x} *_{k} \mu\right)(f)=\int_{\mathbb{R}^{N}} M_{f}(x,|y|) d \mu(y) \quad \text { for all } f \in C_{b}\left(\mathbb{R}^{N}\right) .
$$

Proof. Recall first that $y \mapsto f\left(x *_{k} y\right)$ belongs to $\mathcal{S}\left(\mathbb{R}^{N}\right)$ for $f \in \mathcal{S}\left(\mathbb{R}^{N}\right)$; whence the integral on the right side of (6.1) is well-defined. If $\mu \in M_{k}^{1}\left(\mathbb{R}^{N}\right)$, then for $f \in \mathcal{S}\left(\mathbb{R}^{N}\right)$,

$$
\begin{aligned}
\int_{\mathbb{R}^{N}} f\left(x *_{k} y\right) d \mu(y) & =\int_{\mathbb{R}^{N}} \frac{1}{d_{0}}\left(\int_{S^{N-1}} f\left(x *_{k}|y| \xi\right) w_{k}(|y| \xi) d \sigma(\xi)\right) w_{k}^{-1}(y) d \mu(y) \\
& =\frac{d_{k}}{d_{0}} \int_{\mathbb{R}^{N}} M_{f}(x,|y|)|y|^{2 k} w_{k}^{-1}(y) d \mu(y)=\int_{\mathbb{R}^{N}} M_{f}(x,|y|) d \mu(y) .
\end{aligned}
$$

This shows that the functional $f \mapsto \int_{\mathbb{R}^{N}} f\left(x *_{k} y\right) d \mu(y)$ extends uniquely to a positive bounded Borel measure $\delta_{x} *_{k} \mu \in M_{b}\left(\mathbb{R}^{N}\right)$ and that (6.2) is satisfied. Since $M_{f}=1$ for $f=1, \delta_{x} *_{k} \mu$ is in fact a probability measure.

\section{Theorem 6.3.}

(i) If $\mu \in M_{k}^{1}\left(\mathbb{R}^{N}\right)$, then $P(x, A):=\delta_{x} *_{k} \mu(A)$ defines a k-invariant Markov kernel on $\mathbb{R}^{N}$ with $P(0,)=.\mu$.

(ii) Conversely, if $P$ is a k-invariant Markov kernel with $P(0,.) \in M_{k}^{1}\left(\mathbb{R}^{N}\right)$, then $P(x,)=.\delta_{x} *_{k} P(0,$.$) .$

Proof. For (i), notice first that $\delta_{0} *_{k} \mu=\mu$. Moreover, by the above proposition and equation (4.1),

$$
\begin{aligned}
\left(\delta_{x} *_{k} \mu\right)^{\wedge k}(\xi) & =\int_{\mathbb{R}^{N}} E_{k}(y,-i \xi) d\left(\delta_{x} *_{k} \mu\right)(y)=\int_{\mathbb{R}^{N}} M_{E_{k}(.,-i \xi)}(x,|y|) d \mu(y) \\
& =E_{k}(x,-i \xi) \int_{\mathbb{R}^{N}} j_{\lambda}(|y||\xi|) d \mu(y) .
\end{aligned}
$$


Specializing to $x=0$, this gives

$$
\widehat{\mu}^{k}(\xi)=\int_{\mathbb{R}^{N}} j_{\lambda}(|y||\xi|) d \mu(y),
$$

and thus

$$
\left(\delta_{x} *_{k} \mu\right)^{\wedge k}(\xi)=E_{k}(-i x, \xi) \cdot \widehat{\mu}^{k}(\xi),
$$

which proves that $P$ is $k$-invariant. By Lévy's continuity theorem for the Dunkl transfrom, this also ensures that $x \mapsto \delta_{x} *_{k} \mu$ is continuous with respect to the weak topology on $M^{1}\left(\mathbb{R}^{N}\right)$. This easily implies that $x \mapsto P(x, A)$ is measurable for any $A \in \mathcal{B}\left(\mathbb{R}^{N}\right)$. Thus the proof of (i) is complete. Part (ii) follows immediately from the definition of $k$-invariance and the injectivity of the Dunkl transform.

We also introduce a convolution product for measures from $M_{k}^{1}\left(\mathbb{R}^{N}\right)$ :

Definition 6.4. Let $\mu, \nu \in M_{k}^{1}\left(\mathbb{R}^{N}\right)$. Then $\mu *_{k} \nu \in M^{1}\left(\mathbb{R}^{N}\right)$ is defined by

$$
\mu *_{k} \nu(f):=\int_{\mathbb{R}^{N}} \delta_{x} *_{k} \nu(f) d \mu(x)=\int_{\mathbb{R}^{N}} \int_{\mathbb{R}^{N}} f\left(x *_{k} y\right) d \mu(x) d \nu(y), \quad f \in \mathcal{S}\left(\mathbb{R}^{N}\right) .
$$

Notice that

$$
\left(\mu *_{k} \nu\right)^{\wedge k}=\widehat{\mu}^{k} \widehat{\nu}^{k} \quad \text { for all } \mu, \nu \in M_{k}^{1}\left(\mathbb{R}^{N}\right) .
$$

There is a close relationship between $*_{k}$ on $M_{k}^{1}\left(\mathbb{R}^{N}\right)$ and the convolution $\circ_{\lambda}$ of the Bessel-Kingman hypergroup of index $\lambda=\gamma+N / 2-1$ on $\mathbb{R}_{+}$(cf. Section [2.3). We shall in particular obtain from this connection that $M_{k}^{1}\left(\mathbb{R}^{N}\right)$ is closed w.r.t. $*_{k}$. Let us start with some additional notation.

We denote by $p(\mu) \in M_{b}\left(\mathbb{R}_{+}\right)$the image measure of $\mu \in M_{b}\left(\mathbb{R}^{N}\right)$ under the mapping $x \mapsto|x|$, characterized by

$$
\int_{\mathbb{R}^{N}} f(|x|) d \mu(x)=\int_{0}^{\infty} f(r) d p(\mu)(r) \quad \text { for all } f \in C_{b}\left(\mathbb{R}_{+}\right) .
$$

Further, we write $\mathcal{H}^{\lambda}$ for the Hankel transform of index $\lambda$ on $M_{b}\left(\mathbb{R}_{+}\right)$, i.e.,

$$
\mathcal{H}^{\lambda}(\sigma)(r)=\int_{0}^{\infty} j_{\lambda}(r t) d \sigma(t), \quad \sigma \in M_{b}\left(\mathbb{R}_{+}\right) .
$$

In these terms, (6.3) just states that for $\mu \in M_{k}^{1}\left(\mathbb{R}^{N}\right)$,

$$
\widehat{\mu}^{k}(\xi)=\mathcal{H}^{\lambda}(p(\mu))(|\xi|) .
$$

We also recall from hypergroup theory that the Hankel transfrom $\mathcal{H}^{\lambda}$ is multiplicative w.r.t. the convolution $\circ_{\lambda}$ on $M_{b}\left(\mathbb{R}_{+}\right)$, i.e.,

$$
\mathcal{H}^{\lambda}\left(\sigma \circ_{\lambda} \tau\right)=\mathcal{H}^{\lambda}(\sigma) \mathcal{H}^{\lambda}(\tau) \text { for all } \sigma, \tau \in M_{b}\left(\mathbb{R}_{+}\right) .
$$

Lemma 6.5. $M_{k}^{1}\left(\mathbb{R}^{N}\right)$ is a commutative semigroup with respect to $*_{k}$ with neutral element $\delta_{0}$. The mapping $p:\left(M_{k}^{1}\left(\mathbb{R}^{N}\right), *_{k}\right) \rightarrow\left(M^{1}\left(\mathbb{R}_{+}\right), \circ_{\lambda}\right), \mu \mapsto p(\mu)$, establishes an isometric isomorphism of semigroups as well as a homeomorphism with respect to the weak topologies on both spaces.

Proof. It is obvious that $p$ is an isometric bijection from $M_{k}^{1}\left(\mathbb{R}^{N}\right)$ onto $M^{1}\left(\mathbb{R}_{+}\right)$. Moreover, by Lévy's continuity theorem for the Dunkl transform and the Hankel transform respectively, we deduce from (6.5) that $p$ is a homeomorphism w.r.t. the weak topologies on the stated spaces. Commutativity of $*_{k}$ and the statement on the neutral element are clear. It remains to prove that $M_{k}^{1}\left(\mathbb{R}^{N}\right)$ is closed with 
respect to $*_{k}$, and that $p$ is multiplicative. For this, let $\mu, \nu \in M_{k}^{1}\left(\mathbb{R}^{N}\right)$. Then by (6.4) and (6.5),

$$
\begin{aligned}
\left(\mu *_{k} \nu\right)^{\wedge k}(\xi) & =\widehat{\mu}^{k}(\xi) \widehat{\nu}^{k}(\xi)=\mathcal{H}^{\lambda}(p(\mu))(|\xi|) \mathcal{H}^{\lambda}(p(\nu))(|\xi|) \\
& =\mathcal{H}^{\lambda}\left(p(\mu) \circ_{\lambda} p(\nu)\right)(|\xi|) .
\end{aligned}
$$

Let $\tau \in M_{k}^{1}\left(\mathbb{R}^{N}\right)$ with $p(\tau)=p(\mu) \circ_{\lambda} p(\nu)$. Then the above calculation shows that $\left(\mu *_{k} \nu\right)^{\wedge k}=\widehat{\tau}^{k}$. The injectivity of the Dunkl transform implies that $\mu *_{k} \nu=\tau \in$ $M_{k}^{1}\left(\mathbb{R}^{N}\right)$, as well as

$$
p\left(\mu *_{k} \nu\right)=p(\tau)=p(\mu) \circ_{\lambda} p(\nu) .
$$

This finishes the proof.

Recall that for two Markov kernels $P, Q$ on $\mathbb{R}^{N}$, the composition $P \circ Q$ is defined by

$$
P \circ Q(x, A)=\int_{\mathbb{R}^{N}} Q(z, A) P(x, d z) .
$$

If $P$ and $Q$ are $k$-invariant, then $P \circ Q$ is again $k$-invariant with

$$
((P \circ Q)(x, .))^{\wedge k}(\xi)=P(0, \cdot)^{\wedge k}(\xi) \cdot Q(0, \cdot)^{\wedge k}(\xi) \cdot E_{k}(-i x, \xi) \quad\left(x, \xi \in \mathbb{R}^{N}\right),
$$

cf. 24. A family $\left(P_{t}\right)_{t \geq 0}$ of $k$-invariant Markov kernels on $\mathbb{R}^{N}$ is called a semigroup of $k$-invariant Markov kernels if the following statements hold:

(i) The kernels $\left(P_{t}\right)_{t \geq 0}$ form a semigroup, i.e., $P_{s} \circ P_{t}=P_{s+t}$ for $s, t \geq 0$.

(ii) The mapping $\mathbb{R}_{+} \rightarrow M^{1}\left(\mathbb{R}^{N}\right), t \mapsto P_{t}(0,$.$) , is weakly continuous.$

The above results enable us to construct semigroups of $k$-invariant Markov kernels from convolution semigroups with respect to the hypergroup structure $\circ_{\lambda}$ on $\mathbb{R}_{+}$.

Definition 6.6. A family $\left(\sigma_{t}\right)_{t \geq 0} \subset M^{1}\left(\mathbb{R}_{+}\right)$of probability measures on $\mathbb{R}_{+}$is called a convolution semigroup on $\mathbb{R}_{+}$with respect to $\circ_{\lambda}$, if $\sigma_{s} \circ_{\lambda} \sigma_{t}=\sigma_{s+t}$ for all $s, t \geq 0$ with $\sigma_{0}=\delta_{0}$, and if the mapping $\mathbb{R}_{+} \rightarrow M^{1}\left(\mathbb{R}_{+}\right), t \mapsto \sigma_{t}$, is weakly continuous.

Theorem 6.7. Suppose that $\left(\sigma_{t}\right)_{t \geq 0}$ is a convolution semigroup on $\mathbb{R}_{+}$w.r.t. $\circ_{\lambda}$, and define $\left(\mu_{t}\right)_{t \geq 0} \subset M_{k}^{1}\left(\mathbb{R}^{N}\right)$ by $\mu_{t}:=p^{-1}\left(\sigma_{t}\right) \in M_{k}^{1}\left(\mathbb{R}^{N}\right)$. Then $\mu_{s} *_{k} \mu_{t}=\mu_{s+t}$ for all $s, t \geq 0$, and $P_{t}(x, A):=\delta_{x} \circ_{k} \mu_{t}$ defines a semigroup of $k$-invariant Markov kernels on $\mathbb{R}^{N}$.

Proof. Let $s, t \geq 0$. Then by Lemma 6.5,

$$
p\left(\mu_{s} *_{k} \mu_{t}\right)=\nu_{s} \circ_{\lambda} \nu_{t}=\nu_{s+t}=p\left(\mu_{s+t}\right) .
$$

Since $p$ is injective on $M_{k}^{1}\left(\mathbb{R}^{N}\right)$, this proves that $\mu_{s} *_{k} \mu_{t}=\mu_{s+t}$. Further, the weak continuity of the mapping $t \mapsto \mu_{t}$ on $\mathbb{R}_{+}$is clear from the continuity of $p^{-1}$. According to Theorem 6.3, each $P_{t}$ is a $k$-invariant Markov kernel with $P_{t}(0,)=$. $\mu_{t}$, and

$$
\begin{aligned}
\left(P_{s} \circ P_{t}\right)(x, .)^{\wedge k}(\xi)=E_{k}(-i x, \xi) P_{s}(0, .)^{\wedge k}(\xi) P_{t}(0, .)^{\wedge k}(\xi) & \\
& =E_{k}(-i x, \xi) \widehat{\mu}_{s}^{k}(\xi) \widehat{\mu}_{t}^{k}(\xi)=E_{k}(-i x, \xi) \widehat{\mu}_{s+t}^{k}(\xi)=P_{s+t}(x, .)^{\wedge k}(\xi) .
\end{aligned}
$$

This implies the semigroup property of $\left(P_{t}\right)_{t \geq 0}$. 
Examples 6.8. All the examples for semigroups of $k$-invariant Markov kernels discussed in 24] fit into the concept of Theorem 6.7

(1) ( $k$-Gaussian semigroups). The $k$-Gaussian semigroup $\left(P_{t}^{\Gamma}\right)_{t \geq 0}$ is defined by

$$
P_{t}^{\Gamma}(x, A)=\int_{A} \Gamma_{k}(t, x, y) w_{k}(y) d y \quad(t>0),
$$

with the Dunkl-type heat kernel $\Gamma_{k}$ (cf. (3.1)). It is $k$-invariant and defines a Feller process on $\mathbb{R}^{N}$, the Dunkl-type Brownian motion. According to (3.2) and Lemma 2.2, we may write

$$
\Gamma_{k}(t, x, y)=F_{k}\left(t,-x *_{k} y\right)
$$

with

$$
F_{k}(t, x):=\Gamma_{k}(t, x, 0)=\frac{1}{(2 t)^{\lambda+1} c_{k}} e^{-|x|^{2} / 4 t}
$$

For $t>0$, put

$$
d \mu_{t}(x):=F_{k}(t, x) w_{k}(x) d x \in M_{k}^{1}\left(\mathbb{R}^{N}\right) .
$$

Then $\sigma_{t}:=p\left(\mu_{t}\right) \in M_{\gamma}^{1}\left(\mathbb{R}_{+}\right)$is given by the Rayleigh distribution

$$
d \sigma_{t}(r)=\frac{d_{k}}{(2 t)^{\lambda+1} c_{k}} r^{2 \lambda+1} e^{-r^{2} / 4 t}, \quad t>0 .
$$

It is well known (see e.g. 7.3 .18 of $[5]$ ) that $\left(\nu_{t}\right)_{t \geq 0}\left(\right.$ with $\left.\nu_{0}:=0\right)$ is a convolution semigroup on $\mathbb{R}_{+}$w.r.t. $\circ_{\lambda}$. It defines a Bessel process of index $\lambda$. In order to see that $\left(P_{t}^{\Gamma}\right)_{t \geq 0}$ is associated with $\left(\sigma_{t}\right)_{t \geq 0}$ as in Theorem6.7 it remains to verify that

$$
\delta_{x} *_{k} \mu_{t}=P_{t}^{\Gamma}(x, .) .
$$

But if $f \in \mathcal{S}\left(\mathbb{R}^{N}\right)$, then by Lemma [2.2]

$$
\delta_{x} *_{k} \mu_{t}(f)=\int_{\mathbb{R}^{N}} F_{k}(t, y) f\left(x *_{k} y\right) w_{k}(y) d y=\int_{\mathbb{R}^{N}} \Gamma_{k}(t, x, y) f(y) w_{k}(y) d y .
$$

This yields the claimed identity.

(2) (Subordination). If $\left(P_{t}\right)_{t \geq 0}$ is a semigroup of $k$-invariant Markov kernels and $\left(\rho_{t}\right)_{t \geq 0} \subset M^{1}(\mathbb{R})$ is a convolution semigroup of probability measures on the group $(\mathbb{R},+)$ (in the sense of [4]) which is supported by $\mathbb{R}_{+}$, then a subordinated semigroup $\left(\widetilde{P}_{t}\right)_{t \geq 0}$ of $k$-invariant Markov kernels is defined by

$$
\widetilde{P}_{t}(x, A)=\int_{0}^{\infty} P_{s}(x, A) d \rho_{t}(s) .
$$

Suppose now that $\left(P_{t}\right)_{t \geq 0}$ is associated with a $\circ_{\lambda}$-convolution semigroup $\left(\sigma_{t}\right)_{t \geq 0}$ on $\mathbb{R}_{+}$according to Theorem 6.7. Then it is immediate that $\left(\widetilde{P}_{t}\right)_{t \geq 0}$ is associated in the same way with the $\circ_{\lambda}$-convolution semigroup $\left(\widetilde{\sigma}_{t}\right)_{t \geq 0}$ defined by

$$
\widetilde{\sigma}_{t}:=\int_{0}^{\infty} \sigma_{s} d \rho_{t}(s)
$$

This example in particular includes the $k$-Cauchy kernels in [24]. 


\section{Appendix: Generalized Bessel functions as spherical functions}

For crystallographic reflection groups and certain discrete sets of half-integer multiplicity functions, generalized Bessel functions have an interpretation as the spherical functions of a Euclidean-type symmetric space. This in particular implies a positive product formula as stated in (1.3).

To become more precise, let us first recall the context from [20] and [16]: Suppose that $\mathcal{G}$ is a connected, non-compact semisimple Lie group with finite center. Choose a maximal compact subgroup $\mathcal{K}$ of $\mathcal{G}$, and let $\mathcal{K} \ltimes \mathfrak{p}$ be the Cartan motion group associated with the Cartan decomposition $\mathfrak{g}=\mathfrak{k}+\mathfrak{p}$ of the Lie algebra $\mathfrak{g}$; here $\mathcal{K}$ acts on $\mathfrak{p}$ via the adjoint representation. Choose a maximal abelian subspace $\mathfrak{a}$ of $\mathfrak{p}$, and denote by $\Sigma \subset \mathfrak{a}^{*}$ the roots of $\mathfrak{g}$ with respect to $\mathfrak{a}$. We consider $\Sigma$ as a subset of $\mathfrak{a}$, identifying $\mathfrak{a}$ with its dual via the Killing form $B$, and denote by $G$ the Weyl group of $\Sigma$, acting on the Euclidean space $(\mathfrak{a}, B) \cong\left(\mathbb{R}^{N},\langle.,\rangle.\right)$. Let us consider the spherical functions of the Gelfand pair $(\mathcal{K} \ltimes \mathfrak{p}, \mathcal{K})$ as $\mathcal{K}$-invariant functions on $(\mathcal{K} \ltimes \mathfrak{p}) / \mathcal{K} \cong \mathfrak{p}$. According to Chap. IV of [12], they are of the form $\psi_{\lambda}, \lambda \in \mathfrak{a}_{\mathbb{C}}$, where $\psi_{\lambda} \in C^{\infty}(\mathfrak{p})$ is characterized as the unique $\mathcal{K}$-invariant solution of the joint eigenvalue problem

$$
\partial(p) \psi=p(\lambda) \psi \text { for all } p \in I(\mathfrak{p}), \quad \psi(0)=1
$$

here $I(\mathfrak{p})$ is the space of $\mathcal{K}$-invariants in the symmetric algebra over $\mathfrak{p}$ and $\partial(p)$ is the constant coefficient differential operator associated with $p$. Further, $\psi_{\lambda}=\psi_{\mu}$ iff $\mu=g \lambda$ for some $g \in G$. By taking radial parts of constant coefficient differential operators on $\mathfrak{p}$, it can now be shown that the restriction $\left.\psi_{\lambda}\right|_{\mathfrak{a}}$ (which is $G$-invariant and determines $\psi_{\lambda}$ uniquely) coincides with the generalized Bessel function $J_{k}(., \lambda)$ associated with $G$; the multiplicity function $k$ is given by $k(\alpha)=\frac{1}{4} \sum_{\beta \in \mathbb{R} \alpha \cap \Sigma} m_{\beta}$, with $m_{\alpha}$ the multiplicity of $\alpha \in \Sigma$. A detailed proof of this can be found in [16].

The convolution of $\mathcal{K}$-biinvariant functions on the Cartan motion group can be interpreted as the convolution of a commutative hypergroup structure on the double coset space $(\mathcal{K} \ltimes \mathfrak{p}) / / \mathcal{K} \cong \mathcal{K} \backslash \mathfrak{p}$, which is defined by

$$
\delta_{\mathcal{K} \cdot x} * \delta_{\mathcal{K} \cdot y}=\int_{\mathcal{K}} \delta_{\mathcal{K} \cdot(x+k \cdot y)} d k, \quad x, y \in \mathfrak{p} .
$$

(See [17], Chap. 8 for information about double coset and orbit hypergroups.) If $\mathfrak{a}_{+}$is an arbitrary fixed Weyl chamber of $\mathfrak{a}$, then each orbit $\mathcal{K} \cdot x$ contains a unique $x_{+} \in \overline{\mathfrak{a}_{+}}$, and the mapping $\mathcal{K} \backslash \mathfrak{p} \mapsto \overline{\mathfrak{a}_{+}}, \mathcal{K} \cdot x \rightarrow x_{+}$is a homeomorphism; see 12, Prop. I.5.18. Thus, we obtain an induced hypergroup structure on the closed chamber $\overline{\mathfrak{a}_{+}}$which is determined by the convolution

$$
\delta_{x} * \delta_{y}=\int_{\mathcal{K}} \delta_{(x+k \cdot y)_{+}} d k, x, y \in \overline{\mathfrak{a}_{+}} .
$$

In particular, the $\delta_{x} * \delta_{y}$ are compactly supported probability measures on $\overline{\mathfrak{a}_{+}}$. The identity element of this hypergroup is 0 , and the involution is given by $\left(x_{+}\right)^{-}=$ $(-x)_{+}$. From (7.1) together with the characterization of the spherical functions $\psi_{\lambda}$ by means of their product formula, we see that the set

$$
\left\{\psi \in C\left(\overline{\mathfrak{a}_{+}}\right): \delta_{x} * \delta_{y}(\psi)=\psi(x) \psi(y)\right\}
$$

of continuous, multiplicative functions on the hypergroup $\overline{\mathfrak{a}_{+}}$consists exactly of the functions $\psi_{\lambda}, \lambda \in \mathfrak{a}_{\mathbb{C}}$. With the identification $(\mathfrak{a}, B) \cong\left(\mathbb{R}^{N},\langle.,\rangle.\right), \mathfrak{a}_{+} \cong C$ and 
$J_{k}$ as above, we in particular obtain

$$
J_{k}(x, \lambda) J_{k}(y, \lambda)=\int_{\bar{C}} J_{k}(\xi, \lambda) d\left(\delta_{x} * \delta_{y}\right)(\xi) \quad \text { for all } x, y \in \bar{C}, \lambda \in \mathbb{C}^{N} .
$$

Suppose now that $\mathcal{G}$ is complex. Then $k(\alpha)=1$ for all $\alpha \in R$, and the associated Bessel function $J_{\mathbf{1}}(., \lambda)$ is given by

$$
J_{1}(x, \lambda)=c \sum_{g \in G} \frac{\operatorname{det}(g)}{\pi(x) \pi(\lambda)} e^{\langle x, g \lambda\rangle}
$$

with some constant $c \in \mathbb{C}$; here $\pi$ denotes the fundamental alternating polynomial

$$
\pi(x)=\prod_{\alpha \in R_{+}}\langle\alpha, x\rangle,
$$

cf. [9], Prop. 1.4. (This result remains obviously true if not a single $G$-orbit in $R$, but any $G$-invariant subset, in particular $R$ itself, is considered.) The Laplace representation (2.3) for $E_{k}$ implies that for all $x, \lambda \in \mathbb{R}^{N}$,

$$
J_{\mathbf{1}}(x, \lambda)=\int_{\mathbb{R}^{N}} e^{\langle\xi, \lambda\rangle} d \widetilde{\mu}_{x}(\xi)
$$

with the $G$-invariant probability measure

$$
\widetilde{\mu}_{x}:=\frac{1}{|G|} \sum_{g \in G} \mu_{g x}^{1}
$$

For $y \in \mathbb{R}^{N}$, denote by $\nu_{x, y} \in M^{1}\left(\mathbb{R}^{N}\right)$ the image measure of $\widetilde{\mu}_{x}$ under the translation $\xi \mapsto \xi+y$. Then

$$
\begin{aligned}
& J_{\mathbf{1}}(x, \lambda) J_{\mathbf{1}}(y, \lambda)=c \int_{\mathbb{R}^{N}} \sum_{g \in G} \frac{\operatorname{det}(g)}{\pi(y) \pi(\lambda)} e^{\langle y+\xi, g \lambda\rangle} d \widetilde{\mu}_{x}(\xi) \\
&=c \int_{\mathbb{R}^{N}} \sum_{g \in G} \frac{\pi(\xi)}{\pi(y)} \frac{\operatorname{det}(g)}{\pi(\xi) \pi(\lambda)} e^{\langle\xi, g \lambda\rangle} d \nu_{x, y}(\xi)=\int_{\mathbb{R}^{N}} J_{\mathbf{1}}(\xi, \lambda) \frac{\pi(\xi)}{\pi(y)} d \nu_{x, y}(\xi) .
\end{aligned}
$$

This shows that in the present case, the hypergroup convolution on $\bar{C}$ is given by $\delta_{x} * \delta_{y}=\pi(y)^{-1} \rho_{x, y}$, where $\rho_{x, y}$ is the image measure of $\pi \nu_{x, y}$ under the canonical projection $\mathbb{R}^{N} \rightarrow G \backslash \mathbb{R}^{N} \cong \bar{C}$. Up to a common multiplicative factor, the spherical functions $\psi_{\lambda}$ of $(\mathcal{K} \ltimes \mathfrak{p}, \mathcal{K})$ can be identified with the spherical functions $\Phi_{\lambda}$ of the symmetric space $\mathcal{G} / \mathcal{K}$, cf. [12], Prop. IV.4.10. So the $\Phi_{\lambda}$ satisfy a slightly modified positive product formula, which has recently been investigated in [11]. The representing measures are absolutely continuous with respect to Lebesgue measure in the generic case, and the authors of 11] obtained detailed information on their supports.

\section{ACKNOWLEDGEMENTS}

It is a pleasure to thank J. Scheurle and M. Voit for some valuable comments and discussions. 


\section{REFERENCES}

1. M. Abramowitz and I. A. Stegun, Pocketbook of Mathematical Functions. Verlag Harri Deutsch, Frankfurt/Main, 1984. MR 85j:00005b

2. R. Askey, Orthogonal Polynomials and Special Functions. SIAM, Philadelphia, 1975. MR 58:1288

3. Y. Berest, The problem of lacunas and analysis on root systems, Trans. Amer. Math. Soc. 252 (2000), 3743-3776. MR 2001d:58030

4. C. Berg and G. Forst, Potential Theory on Locally Compact Abelian Groups. Springer-Verlag, 1975. MR 58:1204

5. W. Bloom and H. Heyer, Harmonic Analysis of Probability Measures on Hypergroups. De Gruyter-Verlag, Berlin, 1994. MR 96a:43001

6. C. F. Dunkl, Differential-difference operators associated to reflection groups, Trans. Amer. Math. Soc. 311 (1989), 167-183. MR 90k:33027

7. C. F. Dunkl, Integral kernels with reflection group invariance, Canad. J. Math. 43 (1991), 1213 - 1227. MR 93g:33012

8. C. F. Dunkl, Hankel transforms associated to finite reflection groups, in "Proceedings of the special session on hypergeometric functions on domains of positivity, Jack polynomials and applications," Tampa, 1991, Contemp. Math. 138 (1992), 123-138. MR 94g:33011

9. C. F. Dunkl, Intertwining operators associated to the group $S_{3}$, Trans. Amer. Math. Soc. $\mathbf{3 4 7}$ (1995), 3347-3374. MR 97b:22009

10. C. F. Dunkl and Y. Xu, Orthogonal Polynomials of Several Variables. Cambridge Univ. Press, 2001. MR 2002m:33001

11. P. Graczyk and P. Sawyer, The product formula for the spherical functions on symmetric spaces in the complex case. Pacific J. Math. 204 (2002), 377-393.

12. S. Helgason, Groups and Geometric Analysis. Academic Press, 1984; reprint, Amer. Math. Soc., 2000. MR 86c:22017 MR 2001h:22001

13. L. Hörmander, The Analysis of Linear Partial Differential Operators I. Springer-Verlag, Berlin, 1983. MR 85g:35002a

14. J. E. Humphreys, Reflection groups and Coxeter groups. Cambridge Univ. Press, 1990. MR 92h:20002

15. M.F.E. de Jeu, The Dunkl transform, Invent. Math. 113 (1993), 147-162. MR 94m:22011

16. M.F.E. de Jeu, Dunkl operators, Thesis, Leiden University, 1994.

17. R. I. Jewett, Spaces with an abstract convolution of measures. Adv. Math. 18 (1975), 1-101. MR 52:14840

18. J.F.C. Kingman, Random walks with spherical symmetry, Acta. Math. 109 (1965), 11-53. MR 26:7052

19. H. Mejjaoli and K. Trimèche, On a mean value property associated with the Dunkl Laplacian operator and applications, Integral Transform. Spec. Funct. 12 (2001), 279-302. MR 2003a:33028

20. E. M. Opdam, Dunkl operators, Bessel functions and the discriminant of a finite Coxeter group, Compositio Math. 85 (1993), 333-373. MR 95j:33044

21. M. Rösler, Bessel-type signed hypergroups on $\mathbb{R}$, in: H. Heyer, A. Mukherjea (eds.), Probability measures on groups and related structures XI, Proc. Oberwolfach 1994. World Scientific, Singapore, 1995, 292-304. MR 97j:43004

22. M. Rösler, Generalized Hermite polynomials and the heat equation for Dunkl operators, Comm. Math. Phys. 192 (1998), 519-542. MR 99k:33048

23. M. Rösler, Positivity of Dunkl's intertwining operator, Duke Math. J. 98 (1999), 445-463. MR 2000f:33013

24. M. Rösler and M. Voit, Markov Processes related with Dunkl operators, Adv. Appl. Math. 21 (1998), 575-643. MR 2000j:60019

25. M. Rosenblum, Generalized Hermite polynomials and the Bose-like oscillator calculus, in: Operator Theory: Advances and Applications 73, Birkhäuser-Verlag, Basel, 1994, 369-396. MR 96b:33005

26. G. Szegö, Orthogonal Polynomials. Amer. Math. Soc., New York, 1959. MR 21:5029

27. K. Trimèche, Transformation intégrale de Weyl et théorème de Paley-Wiener associés à un opérateur différentiel singulier sur $(0, \infty)$, J. Math. Pures et Appl. 60 (1981), 51-98. MR 83i:47058 
28. K. Trimèche, Paley-Wiener Theorems for the Dunkl transform and Dunkl translation operators, Integral Transform. Spec. Funct. 13 (2002), 17-38.

29. G. N. Watson, A Treatise on the Theory of Bessel Functions. Cambridge Univ. Press, 1966. MR 96i:33010 (latest reprint)

30. Y. Xu, Integration of the intertwining operator for h-harmonic polynomials associated to reflection groups, Proc. Amer. Math. Soc. 125 (1997), 2963-2973. MR 97m:33004

Mathematisches Institut, Universität Göttingen, Bunsenstrasse 3-5, D-37073 Göttingen, Germany

E-mail address: roesler@uni-math.gwdg.de 